\title{
Solving inverse bioheat problems of skin tumour identification by dynamic thermography
}

\author{
J. Iljaž ${ }^{* 1}$, L. C. Wrobel ${ }^{2}$, T. Gomboc ${ }^{1}$, M. Hriberšek ${ }^{1}$ and J. Marn ${ }^{1}$ \\ 1 - Faculty of Mechanical Engineering, University of Maribor \\ Smetanova 17, SI-2000 Maribor, Slovenia, * corresp: jurij.iljaz@um.si \\ 2 - Brunel University London, Kingston Lane, Uxbridge, UB8 3PH, United Kingdom
}

April 11, 2019

\begin{abstract}
Dynamic thermography is a promising new non-invasive diagnostic technique for skin cancer, not just to identify the skin tumour in its early stage but also to evaluate some of tumour parameters showing the stage and invasiveness. This paper covers the solution of inverse bioheat problems of simultaneous identification of tumour diameter, thickness, blood perfusion rate and thermoregulation coefficient based on the surface temperature difference between healthy skin and lesion during the rewarming period of dynamic thermography. The problem has been treated using numerically generated measurement data for Clark II and Clark IV tumours by adding noise to mimic real measurement data. The solution is based on a more realistic 3D numerical model, composed of different layers including the thermoregulation response of the skin, tumour and surrounding tissue using a deterministic Levenberg-Marquardt optimisation algorithm that is robust and fast. The paper covers the analysis of the starting point of the solution, randomness and level of added noise, as well as the effect of numerical model error on the inverse solution. Tumour diameter and thermoregulation response can be estimated accurately regardless of noise and stage, while blood perfusion and tumour thickness can only be estimated accurately for low noise level or later tumour stage. The solution sensitivity to metabolic heat generation, thickness, blood perfusion rate and thermoregulation coefficient of skin and fat was low, while heat capacity and thermal conductivity of skin and tumour should be determined precisely in the numerical model to be able to evaluate all four tumour parameters as accurately as possible.
\end{abstract}

Keywords: Bioheat, skin tumour, dynamic thermography, inverse problem, temperaturedependent properties, Levenberg-Marquardt algorithm, noise analysis.

\section{Introduction}

In recent years thermography or infrared thermal (IRT) imaging has developed drastically due to the development of infrared (IR) cameras, electronics, technology, computers and numerical modeling, and therefore became a very valuable tool for many applications in 
different fields of science. Thermography is usually used for non-invasive surface temperature measurement based on the emitted thermal radiation from the surface, which is captured by the IR camera. Therefore, it has an advantage over the classical thermocouple measurement when a contactless method over a large area of interest is needed, which is especially useful for monitoring purposes. Elevated temperatures of the human body or changes of the temperature have been connected with diseases for which thermography found its way in medicine for various applications like breast cancer diagnostics, dermatological applications, blood perfusion monitoring, diagnosis of vascular disorder, fever screening, dental diagnostics, diagnosis of thyroid gland disease, eye disease, as well as for therapeutic assessment [1-16]. Thermography in medicine can be done in two ways, passively or actively. Passive or static thermography measures the skin temperature or investigated tissue under a steady-state condition, which is time consuming because the patient has to acclimatise to the conditions in a temperature controlled room [17]. Active or dynamic thermography induces thermal stress by heating or cooling the observed tissue and then measuring the thermal response during the recovery phase, which does not need patient acclimatisation and can reveal more information about the tissue under investigation $[5,6,8,12,15-19]$.

In this paper, we will focus on dynamic thermography for skin tumour detection, as shown by Çetingül and Herman [12]. They carried out a clinical study on 35 patients to show the advantage of dynamic thermography by cooling the skin and observing the recovery phase. By observing the temperature difference between the lesion and healthy skin, they managed to distinguish a malignant melanoma or squamous cell carcinoma from pigmented moles in their early stage. Among all types of skin tumours, malignant melanoma is the most fatal because it metastases rapidly and can quickly spread to soft tissues like lungs and liver [20]. According to Clark et al. [21] and Breslow [22], there is a direct correlation between the survival rate and invasiveness or depth of the tumour. Clark classified skin tumours into five levels from I to V, which is still used nowadays. Clark I represents melanoma in situ, which means that the cells are only in the epidermis or outer layer of the skin. Clark II is located in the papillary dermis layer of the skin, while Clark III is touching the reticular dermis. Clark IV means that the tumour already spread into the reticular or deep dermis, while Clark $\mathrm{V}$ means that it has grown into the fat layer under the skin. For Clark II, the survival rate for malignant melanoma was $72.2 \%$, while for Clark III, IV and V they were $46.5 \%, 31.6 \%, 12.0 \%$, respectively [21]. For this reason, it is important to detect malignant skin tumour in its early stage [12,21-25].

The most frequently used diagnostic technique is visual inspection based on the ABCDE (Asymmetry, Border, Colour, Diameter, Evolution) criteria and dermatoscopes. As it is known, the ABCDE criteria uses only qualitative guidelines for melanoma identification and can therefore produce high rates of false positive or false negative identification. To avoid the risk of missing an early stage melanoma, excisional biopsies are performed for further pathological investigation [25-28]. Therefore, new techniques for skin tumour detection are being developed, which involve a compromise between certain aspects like effectiveness, accuracy, cost and invasiveness, like digital photography, multispectral imaging systems, confocal scanning laser microscopy (CSLM), laser Doppler perfusion imaging (LDPI) optical coherence tomography (OCT), ultrasound and magnetic resonance imaging (MRI) [24, 29-37].

As showed by many authors, dynamic thermography can be a promising diagnostic technique in dermatology for early skin tumour detection $[1-3,5,6,8,12,13,18,35,38]$. Skin 
tumour has higher blood perfusion rate as well as metabolic heat generation $[12,37,39,40]$ from the healthy surrounding tissue, and because the heat transfer in tissue is mostly governed by these two parameters, the tumour will leave a temperature signature, which can be detected using an IR camera. The temperature difference between the lesion and healthy skin is very small and hard to detect using static thermography. Dynamic thermography, on the other hand, by cooling the investigated tissue, will induce higher temperature differences during the rewarming period, which can be more easily observed $[12,41]$. However, dynamic thermography cannot be used just for detection, the taken measurement of temperature during rewarming can be used to evaluate several tumour parameters like size, position, blood perfusion rate, etc., using an inverse problem approach.

There have been many papers on solving inverse bioheat problems of estimating location and size of the tumour [42-45], as well as more specific studies for breast cancer detection [16, 46, 47], skin tumour [48-51] and blood perfusion estimation [44, 52-54]. However, many of them still use simplified 2D or 3D numerical models composed only of tumour and surrounding tissue $[42,43,45,48]$. Luna et al. [48] used a simplified 2D numerical model to identify thickness and blood perfusion rate of the skin tumour based on a steady-state surface temperature using Simulated Annealing (SA) algorithm. Bhowmik and Repaka [49] upgraded the problem by using a 3D multilayer model of the skin to estimate four tumour parameters; thickness, diameter, blood perfusion rate and metabolic heat generation, by Genetic Algorithm (GA) and SA using steady-state temperature information. Bhowmik et al. $[55,56]$ also made some studies about how difficult it is to estimate the position and size of the tumour around thermally significant blood vessels using static and dynamic thermography or FMTWI (Frequency Modulated Thermal Wave Imaging). Like Bhowmik and Repaka [49], we successfully estimated four tumour parameters using temperature information from dynamic thermography using DOE (Design of Experiment) [38] where we also investigate the difference between static and dynamic thermography and why dynamic thermography can give us more information about the investigated tissue.

The solution of the inverse problem strongly depends on the measurement noise and the accuracy of the numerical model based upon the estimation of unknown parameters is made. Simplified numerical models cannot give a realistic estimation of searched variables because the simulated data do not follow the measurement data accurately. Therefore, a great effort should be put in making the numerical model as realistic as possible by including the thermoregulation response of the skin, tumour and muscle during dynamic thermography, as described in our previous work [41]. Therefore, this paper present the possibility to estimate four tumour parameters; diameter, thickness, blood perfusion rate and thermoregulation coefficient (response), using non-invasive dynamic thermography based on a more realistic 3D numerical model. For now, the inverse bioheat problem is investigated and solved numerically, representing a base for further research and experimental work. To solve the inverse problem we used the Levenberg-Marquardt algorithm, as optimisation, combined with direct numerical simulation using a 3D multilayer model including the thermoregulation response of blood perfusion and metabolic heat generation as described in [41]. The problem is solved based on the surface temperature information or temperature difference between the tumour and healthy skin during the rewarming period, that has been generated numerically. We tested the inverse analysis algorithm for two different test cases; Clark II and Clark IV tumours, to evaluate how the tumour 
stage affects the evaluation of the parameters. However, to mimic real measurement data, noise has been added to the generated temperature response, to analyse how the inverse solution is affected by noise. The paper also covers how the model error or uncertainty of some parameters affects the solution, and which of them should be determined more accurately for a precise parameter estimation.

Therefore, the novelty of this paper can be found in using a more realistic 3D multilayer tissue model including the thermoregulation response to identify four skin tumour parameters based on dynamic thermography measurements, especially the feasibility of estimating the thermoregulation coefficient of the skin tumour, which has not been estimated before. The novelty can be also found in a more detailed analysis of the inverse problem for the estimation of four skin tumour parameters, regarding the measurement noise and model error.

The paper is organized as follows: Section 2 presents the inverse bioheat problem with the numerical model described by the governing equation, thermoregulation model and boundary conditions, and the Levenberg-Marquardt optimization algorithm. Section 3 discusses the test examples for Clark II and IV tumours, material properties, computational mesh, etc., to simulate dynamic thermography together with a description of measurement data and model error. Section 4 discusses the solution of the inverse problem using different starting points and level of measurement noise. The paper closes with Section 5 that summarizes this work and emphasizes the importance of its results.

\section{Inverse bioheat problem}

Direct problems are used when the numerical simulation of certain process or phenomena is needed. However, when unknown variables, material properties, shape or mathematical model are needed and cannot be determined using direct measurement or observation, we are dealing with inverse problems, which are solved based on the proposed mathematical and numerical model and indirect measurement data. The difference between direct and inverse problems is that, for direct problems, the numerical model composed from governing equations, computational domain, boundary conditions, material properties, etc., is well defined and known, while for inverse problems we are trying to identify or estimate the missing model parameters which are not exactly determined. Here, the accuracy of estimated parameters strongly depends on the proposed model that describes the observed phenomena or process, its error and the measurement noise.

Because the inverse solution depends on the mathematical and numerical models adopted, the most common approach is to initially solve a direct problem with guessed unknown parameters and then comparing the solution of the direct problem with the measurement data. To minimize the number of guesses, optimisation techniques are used, minimizing the difference between the direct problem solution and measurement data, and the optimum represents the solution of the inverse problem $[38,44,46,48,49,54]$. Therefore, inverse problems are difficult to solve and usually ill-posed, which means that the solution of the inverse problem will not converge when there is noise in the measurement data or the numerical model does not follow the observed process. Usually, for ill-conditioned, problems we have to impose a regularization or damping technique to stabilize the solution $[52,54]$.

Inverse problems can be practically applied everywhere and therefore, they found 
their way in medical diagnostic as well. The idea of detecting location, size and stage of the tumour, cancerous tissue or other vascular diseases using information of abnormal temperature or heat flux on the skin surface is not new $[4,14,45,50]$. The tissue temperature is mainly controlled by the blood perfusion rate, metabolic heat generation and heat transfer with the surrounding environment. If the tissue temperature changes, this can indicate an abnormal pathological or physiological state of the tissue under the same environmental conditions. It is known that cancerous tissue or tumours have elevated blood perfusion rate and metabolic heat generation $[12,37,40]$, which reflects in elevated tissue temperature on the skin surface, depending on the location and its stage [45, 46, 49-51]. To estimate the position, size and stage of the tumour, a non-invasive technique based on the skin surface temperature is very appealing and promising nowadays due to the development of IR cameras, numerical methods and computers. A very popular problem using this technique is breast cancer detection, which is still under development by many researchers $[1,3,14-16,46]$. As already described, in this paper we will apply an inverse bioheat problem to skin tumour identification using dynamic thermography, that showed many advantages over the static one $[8,12,13,38,41]$.

The size of the tumour and its blood perfusion rate can be easily obtained using the dynamic approach, even for an early stage tumour and noisy data, while the tumour metabolic heat generation would be hard to estimate due to its low sensitivity $[12,38,41$, 49]. In our previous paper [41], we introduced a new numerical model for skin tumour tissue including the local thermoregulation response of the skin and tumour, imposing the blood perfusion rate and metabolic heat generation as temperature-dependent variables. The thermoregulation response plays its role in dynamic thermography and has an effect on the temperature difference between the tumour and healthy skin during the recovery phase. Because there is so little information about the blood perfusion thermoregulation for the tumour, especially for cooling, we assumed the value of the thermoregulation coefficients $Q_{10, b}$ and $Q_{10, m}$ and modeled an almost linear response. However, dynamic thermography can help us not only to estimate the size and blood perfusion rate of the tumour but also to get information about the thermoregulation response.

This paper tries to estimate four skin tumour parameters that are important from the diagnostic point of view, to identify the stage and invasiveness of the tumour, using only non-invasive measurements of skin surface temperature by dynamic thermography. These parameters are diameter and thickness of the tumour, tumour blood perfusion rate and thermoregulation response coefficient, which represents the novelty of this paper. Tumour shape is mostly irregular as well as due to the vascularity they are non-homogeneous, for which the geometrical parameters represent the approximated shape of the tumour with the cylinder, while physiological parameters represents the mean values for the investigated lesion that are important in diagnostic. In our previous work [38], we discussed the difference between using static and dynamic thermography for tumour parameter estimation, but now we replace the metabolic heat generation by the thermoregulation coefficient due to its low sensitivity. From the diagnostic point of view, the most important parameters are blood perfusion rate and thickness of the tumour, which reflect its invasiveness, however the information about the thermoregulation response of the tumour or its thermoregulation coefficient can help us in understanding its physiology and also the potential of monitoring the effectiveness of new drugs.

This paper presents the numerical background for solving inverse bioheat problems and the feasibility of making dynamic thermography as a skin tumour screening technique, 
covering the numerical model based on which the estimation of four unknown parameters can be made. Therefore, the problem is solved using numerically generated measurement data with known values of the searched parameters, to be able to evaluate how the model uncertainty and measurement noise affect the inverse solution, which is also a novelty of this paper. By adding different levels of noise to the measurement data, we are able to estimate the sensitivity of the inverse problem solution which is needed to evaluate the errors when real measurement data are used, as well as by changing different model parameters due to uncertainty or data noise, we are able to analyze which model parameters are the most important for an accurate estimation of the searched parameters.

\section{$2.1 \quad$ Numerical model}

This paper adopts a non-homogeneous 3D model of the skin, composed of epidermis, papillary dermis, reticular dermis, fat, muscle and a tumour in the skin layers, as shown in Figure 1. The model also includes the thermoregulation response of the skin, tumour and other tissues to predict the skin temperature during the recovery phase of dynamic thermography as accurately as possible. The base for the non-homogeneous model has been taken from Çetingül and Herman [12,39], Cheng and Herman [18] and Bhowmik and Repaka [49], while the base for the tissue thermoregulation response has been taken from Silva et al. [57] and Fiala et al. [58,59]. A more detailed description of the model can be found in our previous work [41], however, we will present only the most important aspects of the model in this paper.

Çetingül and Herman [39] concluded that the actual shape of the lesion does not affect the temperature response on the skin surface during the rewarming period and that the most important tumour parameters are average volume and thickness. Therefore, in this model, we use a cylindrical representation of the tumour, described by diameter and thickness. Even if the actual shape of the tumour is not cylindrical, diameter and

a)

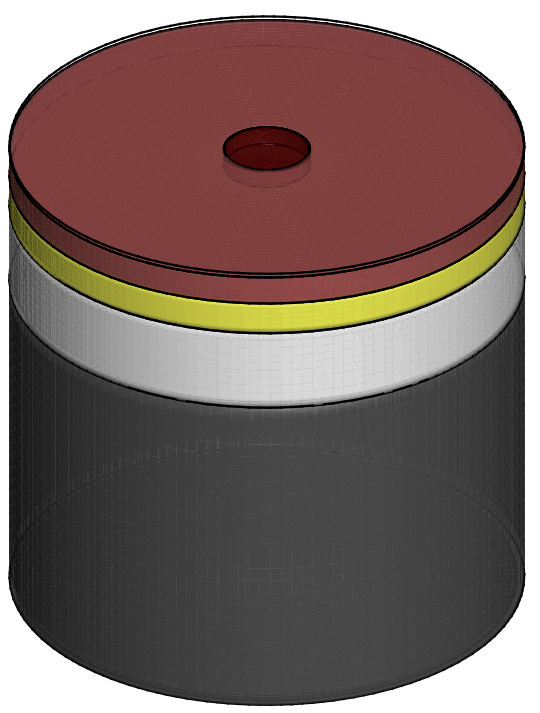

b)

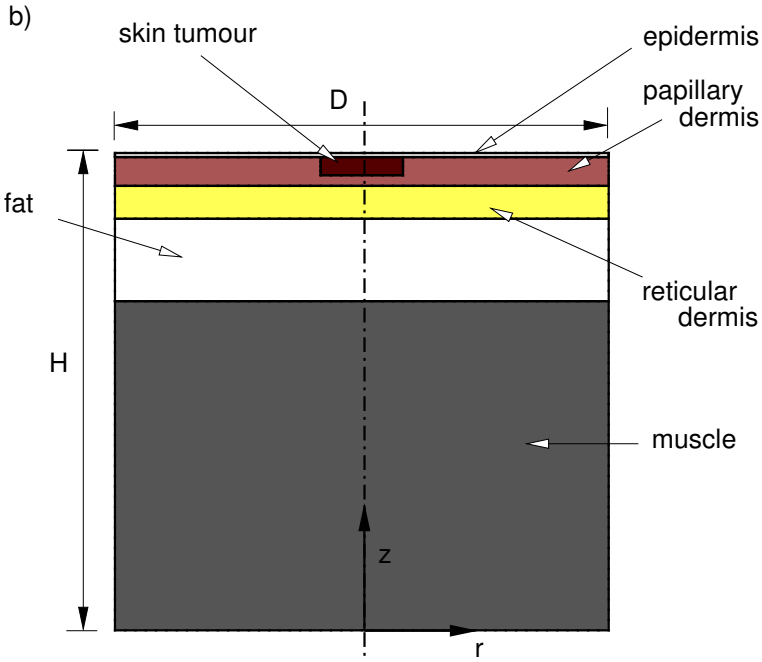

Figure 1: Computational domain of the numerical model including skin tumour: a) isometric view and b) cross-sectional view with notation. 
thickness represents the average value of the non-symmetrical tumour approximated with the cylinder, which is still important to evaluate the stage or invasiveness of the skin tumour. To make the modelling easier and to reduce the computational time the whole computational domain has been modelled using a cylindrical shape, as can be seen in Figure 1.

To describe heat transfer in a biological tissue, we use Pennes' bioheat model [60], which is widely accepted $[15,18,19,39,46,49,50,57]$ and is written as:

$$
\rho c_{p} \frac{\partial T}{\partial t}=\vec{\nabla} \cdot(\lambda \vec{\nabla} T)+\omega_{b} \rho_{b} c_{p, b}\left(T_{a}-T\right)+q_{m},
$$

where $T=T(\vec{r}, t)$ represents tissue temperature, $\rho, \lambda$ and $c_{p}$ are the effective tissue density, thermal conductivity and specific heat, respectively, $\omega_{b}$ is blood perfusion rate, $\rho_{b}$ blood density, $c_{p, b}$ is specific heat of the blood, $T_{a}$ is arterial blood temperature, $t$ time and $q_{m}$ metabolic heat source. The blood perfusion rate is a scalar representing the volumetric blood flow rate per volume of the tissue through small arterioles and capillary bed. Pennes assumed that heat transfer between the blood flow and surrounding tissue happens on the capillary level due to the large interfacial area, therefore, the blood perfusion term acts like a heat source or sink depending on the temperature difference between tissue and arterial blood flow. During the cooling process in dynamic thermography, blood perfusion acts like a heat source, heating up the tissue during thermal recovery, similar to the metabolic heat source which depends on cell activity. Between these two effects, blood perfusion plays a major role in reheating the tissue.

Material properties and other parameters in equation (1) are usually treated as constant due to the lack of an accurate mathematical model to describe the changing mechanism. These parameters are also estimated because of the lack of measurement data or because of the large deviation from various authors [39,61, 62]. However, because it is known that blood perfusion rate and metabolic heat generation of skin, muscle and other tissues are regulated by central and local thermoregulation $[58,59]$, we include a local thermoregulation model to simulate the cooling effect of dynamic thermography more accurately. Therefore, metabolic heat generation and blood perfusion rate for each tissue have been modelled using the van't Hoff effect [57-59,62-64] as:

$$
q_{m}(T)=q_{m, b a s} Q_{10, m}^{\left(\frac{T-T_{0}}{10}\right)},
$$

where $q_{m, \text { bas }}$ represents the basal metabolic rate at rest, $Q_{10, m}$ the metabolic rate coefficient and $T_{0}$ is the equilibrium temperature of the body, and:

$$
\omega_{b}(T)=\omega_{b, b a s} Q_{10, b}^{\left(\frac{T-T_{0}}{10}\right)},
$$

where now $Q_{10, b}$ represents the blood perfusion rate coefficient, which is usually the same as $Q_{10, m}$ [57], and $\omega_{b, b a s}$ represents the basal blood perfusion rate. All other parameters and material properties in Pennes equation (1) such as density, specific heat, thermal conductivity, etc., have been treated as constant for each layer.

To simulate dynamic thermography or the cooling/rewarming process of the skin, we have to prescribe appropriate initial and boundary conditions for the computational domain. Due to the cylindrical shape of the domain, we introduce a cylindrical coordinate 
system $\vec{r}=\vec{r}(r, \varphi, z)$ to simplify the notation. For the bottom part, we prescribe the constant body core temperature $T_{0}$, assuming that the muscle tissue is thick enough, as:

$$
T(r, \varphi, z, t)=T_{0}, \quad 0 \leq r \leq D / 2, \quad z=0, \quad 0 \leq t \leq \tau,
$$

where $D$ is the diameter of the computational domain and $\tau$ represents the simulation time including the cooling process of dynamic thermography. On the sides of the computational domain, we prescribe adiabatic boundary conditions assuming that the diameter $D$ of the computational domain is large enough not to affect the computational solution:

$$
\frac{\partial T(r, \varphi, z, t)}{\partial r}=0, \quad r=D / 2, \quad 0 \leq z \leq H, \quad 0 \leq t \leq \tau,
$$

where $H$ represents the total height of the computational domain. At the skin surface, we have to prescribe the cooling and rewarming processes. For the cooling process, a constant temperature has been chosen due to the deep penetration and high temperature contrast during the rewarming period [18], while for the rewarming period the heat transfer between the surrounding environment and the skin has been modeled using a Robin boundary condition. Therefore, for the skin surface, the following boundary condition has been prescribed:

$$
\begin{gathered}
T(r, \varphi, z, t)=T_{c}, \quad 0 \leq t \leq t_{c} \\
\lambda \frac{\partial T(r, \varphi, z, t)}{\partial z}=\alpha\left[T(r, \varphi, z, t)-T_{\infty}\right], \quad t_{c}<t \leq \tau, \\
0 \leq r \leq D / 2, \quad z=H,
\end{gathered}
$$

where $T_{c}$ represents the cooling temperature, $t_{c}$ the cooling time period, $\alpha$ represents the heat transfer coefficient and $T_{\infty}$ the surrounding temperature. The heat transfer coefficient can include many effects such as heat convection, thermal radiation and water evaporation $[49,57,59]$. However, thermal radiation is negligible in this case due to the small temperature difference between the skin and surrounding environment, as well as water evaporation by sweating because the cooling-rewarming process does not induce sweating. The main contribution during the rewarming period is heat convection with the surrounding air, that is not intense as the skin rewarms mostly because of the blood perfusion rate or internally generated heat. For all described boundary conditions the angle $\varphi$ varies from $0 \leq \varphi \leq 2 \pi$ and was omitted in the above description for a clearer presentation. For the initial temperature condition $T(r, \varphi, z, t=0)$, we prescribe the steady-state solution of the bioheat problem determined with the boundary conditions (4), (5) and the rewarming part of condition (6). To solve the transient bioheat problem, simulating the dynamic thermography defined by equation (1), corresponding models (2) and (3) and boundary conditions (4)-(6), we also have to impose equilibrium and compatibility conditions on the interface between two layers. The compatibility condition is $T_{l}=T_{l+1}$ and equilibrium condition $\lambda_{l} \partial T_{l} / \partial n_{l}=\lambda_{l+1} \partial T_{l+1} / \partial n_{l+1}$, where $n$ represents the normal on the interface and indices $l$ and $l+1$ represent adjoining tissues. These two conditions across interfaces impose continuity of the temperature and heat flux.

To solve the inverse bioheat problem a fast and numerically accurate solver is needed, especially when solving an inverse problem identifying several parameters, because the number of model evaluations rapidly increases with the number of parameters and the computational time can go into days and months. Because of the shape of the computational domain and adiabatic boundary conditions on the sides, the solution of the 
transient bioheat transfer is 3D-axisymmetrical, which means that the solution does not depend on the angle $\varphi$, therefore $T=T(r, z, t)$. For this reason, we can reduce the problem size by using only a $2 \mathrm{D}$ cross-sectional mesh. To solve the direct bioheat problem using described numerical model, a subdomain BEM (Boundary Element Method) approach using a 3D-axisymmetrical elliptic fundamental solution has been used, as described in our previous work [41]. The solver is fast, accurate and adequate to tackle the inverse problem presented in this paper.

\subsection{Optimisation algorithm}

The inverse bioheat problem tackled in this paper is to estimate four tumour parameters (diameter, thickness, blood perfusion rate and thermoregulation coefficient) based on the skin temperature response during the rewarming period of dynamic thermography. The observation variables upon which the estimation is made is surface temperature difference between the tumour and the healthy skin during the recovery phase. Therefore, during the cooling process it is needed to cool down the lesion and a surrounding healthy skin upon which the temperature difference is evaluated. The advantage of using the temperature difference and not the absolute temperature is to avoid the error of absolute temperature measurements, as well as to reduce the error made by prescribed model boundary conditions. As we already suggest in our previous paper [41], observing the temperature difference during dynamic thermography is more appropriate than observing the absolute temperature, because the temperature difference carries the information about the underlying tumour, as observed by many authors [12, 46, 48-50].

We used an optimisation approach to solve the inverse problem by introducing an objective function, which is minimized during the optimisation process. The objective function for this inverse problem is defined as:

$$
F(\vec{y})=\sum_{t=1}^{n_{t}} \sum_{p=1}^{n_{p}}\left(\Delta T_{s, p, t}(\vec{y})-\Delta T_{m, p, t}\right)^{2}=\vec{f}^{t r}(\vec{y}) \cdot \vec{f}(\vec{y}),
$$

where $F(\cdot)$ represents the value of the objective function, $\vec{y}$ is the vector of unknown parameters, $\Delta T_{s}$ and $\Delta T_{m}$ stand for the simulated and measured skin surface temperature difference during the rewarming period, respectively, $t$ and $p$ stand for time and location of the temperature measurement or simulated response, $n_{t}$ and $n_{p}$ are the number of observed data in time and the number of measurement points, respectively, $\vec{f}(\cdot)$ represents the residual vector; $\vec{f}(\vec{y})=\Delta T_{s}(\vec{y})-\Delta T_{m}=\left\{f_{i} ; i=1, m\right\}$, where $m=n_{t} n_{p}$, and $t r$ stands for transpose. Vector $\vec{y}$ is defined as $\vec{y}=\vec{y}\left(d, h, \omega_{b}, Q_{10}\right)=\left\{y_{j}, j=1, n\right\}$, where $n=4, d$ represents the diameter of the tumour, $h$ its thickness, $\omega_{b}$ the blood perfusion rate and $Q_{10}=Q_{10, m}=Q_{10, b}$ the thermoregulation coefficient of the tumour, while $y_{j}$ represents the parameter $j$ in general. As can be seen, the objective function reflects the difference between the direct problem solution for a given set of searched parameters and the measurement data. When the minimum of the objective function is found, the solution to a given inverse problem is obtained. For the inverse problem, the objective function has to have only one global minimum, otherwise the solution is not unique and the evaluation of parameters is not possible $[38,49]$. The skin surface temperature response of dynamic thermography is enough to give us a unique solution for four different parameters even for noisy measurement data, while using static thermography is not [38]. 
We use a deterministic optimisation algorithm, because the objective function for dynamic thermography is smooth [38], as well as they are faster from stochastic optimization techniques like GA (Genetic Algorithm), PSO (Particle Swarm Optimisation) or SA (Simulated Annealing) due to the lower number of direct problem evaluations. The optimisation technique used in this paper is the Levenberg-Marquardt (LM) algorithm, which is a combination of steepest descent and Gauss-Newton optimisation. In our previous paper [52] where space dependent blood perfusion estimation has been done, the LM algorithm proves to work better than BFGS (Broyden-Fletcher-Goldfarb-Shanno) algorithm, was faster and not sensitive to the initial guess, which is the reason for choosing it. We could also use more basic deterministic optimisation like SD (Steppes Descent) or CG (Conjugate Gradient) method which are of first-order and have linear convergence rate, however the convergence rate of LM algorithm is superlinear near the solution making the optimisation process faster. In general, optimisation can be written as:

$$
\text { find } \quad \vec{y}^{*}=\operatorname{argmin}_{\vec{y}}[F(\vec{y})] \text {, }
$$

where $\vec{y}^{*}$ represents the minimum of the objective function or solution of the inverse problem, while for a deterministic approach a better solution is found as:

$$
\vec{y}_{k+1}=\vec{y}_{k}+\beta_{v} \vec{s}_{k} \Rightarrow F\left(\vec{y}_{k+1}\right)<F\left(\vec{y}_{k}\right),
$$

where $\vec{s}$ represents the search direction, $\beta$ a step size and indices $k$ and $v$ stand for iteration and trial step, respectively. Each approach defines the search direction and step size in its unique way. LM algorithm uses the linear Taylor expansion of the residual vector $\vec{f}\left(\vec{y}_{k}+\vec{s}_{k}\right)$, that gives a search direction as the solution to the equation system [52]:

$$
\left([J]_{k}^{t r} \cdot[J]_{k}+\mu_{k}[I]\right) \vec{s}_{k}=-[J]_{k}^{t r} \cdot \vec{f}\left(\vec{y}_{k}\right)
$$

where $[J]$ represents the Jacobian matrix, $\mu$ is a damping parameter and $[I]$ the identity matrix. As seen from equation (10) the search direction is evaluated at each iteration step, together with the Jacobian matrix and damping parameter. The Jacobian matrix is evaluated numerically using first-order finite difference scheme as:

$$
J_{i, j}=\frac{\partial f_{i}}{\partial y_{j}} \approx \frac{f_{i}\left(\vec{y}+\Delta y_{j}\right)-f_{i}(\vec{y})}{\Delta y_{j}},
$$

where indices $i$ and $j$ represent the row and column of matrix $[J]$, respectively, or residual data $i$ and parameter $j$, and $\Delta y_{j}$ represents the change of parameter $j$, which has been taken as $1 \%$ of its value; $\Delta y_{j}=0.01 y_{j}$. To evaluate the Jacobian matrix and to calculate the new search direction at each iteration step $k$, we have to make four evaluations of the direct problem, changing the value of the unknown parameters separately. This is the cost of using this approach, because the search direction is based on the gradient of the objective function that we need to evaluate.

Once the search direction is obtained from equation (10), we can update the solution by using equation (9) and check the descent criteria; $F\left(\vec{y}_{k+1}\right)<F\left(\vec{y}_{k}\right)$. For the first trial the step size is taken as $\beta_{0}=1.0$, because the search direction is also controlled by the damping parameter $\mu$. If the descent criteria is not met, the step size is then reduced by $\beta_{v+1}=\beta_{v} / 2$. 
The damping parameter is calculated at each iteration as [52]:

$$
\mu_{k+1}=\mu_{k} \max \left[\frac{1}{3}, 1-\left(2 \theta_{k}-1\right)^{3}\right]
$$

where $\theta$ represents the gain ratio defined as:

$$
\theta_{k}=\frac{F\left(\vec{y}_{k}\right)-F\left(\vec{y}_{k+1}\right)}{Z(0)-Z\left(\beta_{v} \vec{s}_{k}\right)}
$$

where $Z(\cdot)$ represents a linear Taylor expansion of the objective function. For the first iteration step the damping parameter has been chosen as $\mu_{0}=10^{-5} \max \left[[J]^{t r} \cdot[J]\right]$. If the damping parameter is high, the LM method will calculate the search direction close to the steepest descent where the absolute value of the search direction will be small, therefore there is no need to apply optimum step size searching. However, if the damping parameter is low, the search direction will be close to the Gauss-Newton direction and convergence will be much faster.

To stop the optimisation algorithm, we used three stopping criteria where only one of them has to be fulfilled [52];

$$
\begin{gathered}
k>k_{\max }, \\
\left\|[J]_{k}^{t r} \cdot \vec{f}\left(\vec{y}_{k}\right)\right\|_{\infty} \leq \varepsilon_{1}, \\
\left\|\vec{y}_{k+1}-\vec{y}_{k}\right\| \leq \varepsilon_{2}\left(\left\|\vec{y}_{k}\right\|+\varepsilon_{2}\right),
\end{gathered}
$$

where $k_{\max }$ represents the maximum number of iterative steps and $\varepsilon_{1}$ and $\varepsilon_{2}$ the tolerance for the gradient and step size, respectively. The first stopping criteria is met when the number of iterative steps is greater than allowed, while the second one represents the minimum value of gradient and is met when we are in the proximity of the optimum, and the third one the minimum step size. The tolerance for the second and third criteria has been taken as $\varepsilon_{1}=\varepsilon_{2}=10^{-7}$.

\section{Computational examples}

The solution of inverse bioheat problems is based on the non-homogeneous numerical model of the skin tissue including the thermoregulation response of blood perfusion and metabolic heat generation of different layers, using LM optimisation technique. However, to test the inverse solution, we need measurement data, which have been generated numerically for Clark II and Clark IV tumours, solving a direct problem using a numerical model described in [38] and by adding different levels of noise to mimic realistic temperature measurements on the skin surface. The reason to test the inverse problem solution on numerically generated data is to be able to analyse the effect of measurement noise or model error on the solution, because the exact value of the searched parameters is known. Therefore, the inverse problem will be tested for two cases, Clark II and Clark IV, presenting the early and later stage of the skin tumour $[12,21,22]$, to show the feasibility of estimating all four parameters of the tumour simultaneously.

Material properties and dimensions for each layer and different tumour sizes used in the numerical model are presented in Table 1. Clark II size $(d=2.0 \mathrm{~mm}, h=0.44 \mathrm{~mm})$ presents a tumour embedded in the papillary dermis, while Clark IV $(d=2.5 \mathrm{~mm}, h=$ 
$1.1 \mathrm{~mm}$ ) protrudes into the reticular dermis layer. The material properties, layer thickness and tumour sizes have been chosen based on a literature review [12, 18, 21, 22, 39, 49] and found to be appropriate to test our inverse problem. Regarding the thermoregulation response of the different layers, the thermoregulation coefficient $Q_{10, m}=Q_{10, b}=2.0$ has been chosen for skin layers and muscle [57-59], representing exponential behavior of the metabolic heat generation and blood perfusion rate, while for the tumour a more linear behavior is expected and the value of $Q_{10, m}=Q_{10, b}=1.1$ has been prescribed [41]. The mean body core temperature for a healthy person in resting position is in the range of $36.5^{\circ} \mathrm{C}-37.5^{\circ} \mathrm{C}$, therefore the arterial blood temperature $T_{a}$, as well as the body core temperature at equilibrium $T_{0}$ has been set to $T_{a}=T_{0}=37.0^{\circ} \mathrm{C}[12,39,49]$.

To simulate dynamic thermography we have to prescribe the cooling/rewarming procedure, defined by boundary condition (6) prescribing cooling time, temperature and rewarming stage. Different authors prescribed different cooling times and temperatures $[12,18,19,39,49]$. For this paper, the cooling temperature and duration have been taken from Bhowmik and Repaka [49] and Çetingül and Herman [12], due to a good penetration depth and thermal response, and are $t_{c}=60 \mathrm{~s}$ and $T_{c}=13^{\circ} \mathrm{C}$. As for the surrounding conditions, the ambient temperature has been chosen to be $T_{\infty}=22.4^{\circ} \mathrm{C}[12,49]$ and the heat transfer coefficient $\alpha=10 \mathrm{~W} / \mathrm{m}^{2} K[12,18]$. We simulate $10 \mathrm{~min}=600 \mathrm{~s}$ of rewarming period which is long enough. The biggest temperature difference between the tumour and surrounding tissue appears up to $60 \mathrm{~s}$ after the end of cooling process and then decline towards a steady-state condition [18]. Therefore, the total time to simulate the whole dynamic thermography test has been set to $\tau=660 \mathrm{~s}$. To solve the inverse bioheat problem we only need information about the skin surface temperature during the rewarming period, nevertheless we have to simulate the whole process to accurately determine the tissue response.

In our work $[38,41,65]$, we used a structured mesh with a representative element size of $\Delta r=0.5 \mathrm{~mm}$ and constant time step $\Delta t=1 \mathrm{~s}$. However, for this paper, we upgraded the computational mesh using different mesh density for tumour and surrounding tissue, as well as the time discretization using an adaptive time step. During the fast temperature change a small time step is used to capture the transient change (at the beginning of the cooling and rewarming process), while for slow temperature change the time step has been increased. To assure numerical accuracy of the direct problem, we carried out a mesh and time discretization analysis. The aim is to reduce the computational time of

\begin{tabular}{c|cc|ccccc} 
Material & $d[\mathrm{~mm}]$ & $h[\mathrm{~mm}]$ & $\rho\left[\mathrm{kg} / \mathrm{m}^{3}\right]$ & $c_{p}[\mathrm{~J} / \mathrm{kgk}]$ & $\lambda[\mathrm{W} / \mathrm{mK}]$ & $\omega_{b, \text { bas }}[1 / \mathrm{s}]$ & $q_{m, \text { bas }}\left[\mathrm{W} / \mathrm{m}^{3}\right]$ \\
\hline \hline epidermis & - & 0.1 & 1200 & 3589 & 0.235 & 0.0 & 0.0 \\
papillary dermis & - & 0.7 & 1200 & 3300 & 0.445 & 0.0002 & 368.1 \\
reticular dermis & - & 0.8 & 1200 & 3300 & 0.445 & 0.0013 & 368.1 \\
fat & - & 2.0 & 1000 & 2674 & 0.185 & 0.0001 & 368.3 \\
muscle & - & 8.0 & 1085 & 3800 & 0.510 & 0.0027 & 684.2 \\
\hline tumour Clark II & 2.0 & 0.44 & 1030 & 3852 & 0.558 & 0.0063 & 3680 \\
tumour Clark IV & 2.5 & 1.1 & 1030 & 3852 & 0.558 & 0.0063 & 3680 \\
\hline blood & - & - & 1060 & 3770 & - & - & -
\end{tabular}

Table 1: Material properties and tissue dimensions [12, 18, 39, 49]. 

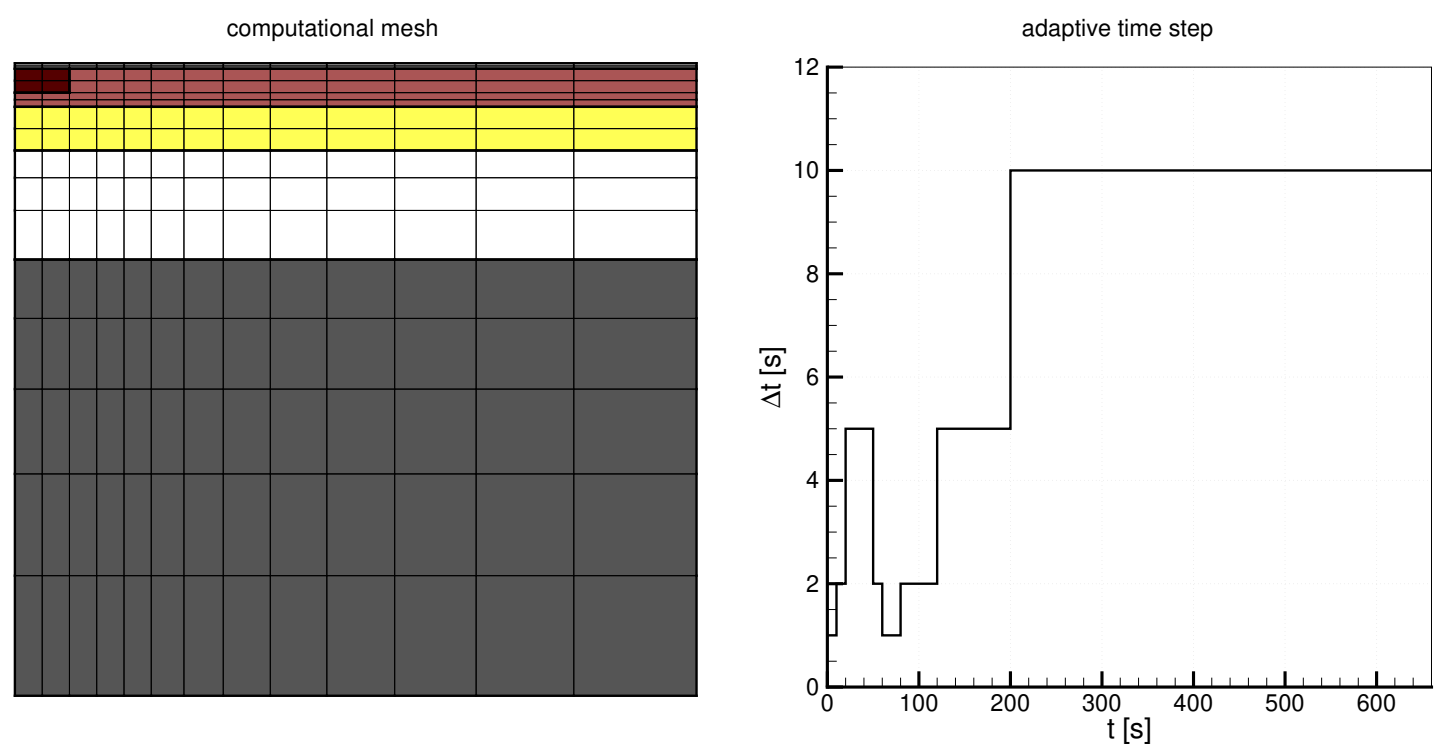

Figure 2: Representative computational mesh for Clark II tumour and adaptive time step selection.

the direct problem, which is very important for inverse or optimisation problems. The final 2D computational mesh of the revolving cross section and selection of the adaptive time step for the direct problem is shown in Figure 2. While for the domain size, we decided to used a domain size of $D=25 \mathrm{~mm}$, to assure the solution is not affected by the adiabatic boundary condition (5).

\subsection{Measurement data}

To evaluate geometric and physiological parameters of the tumour, the temperature difference on the skin surface between the lesion and surrounding healthy skin during the rewarming process is needed. The measurement data at dynamic thermography is obtained by the IR camera recording of the absolute surface temperature at the lesion region together with the surrounding healthy skin. Therefore, to obtain the data needed for solving presented inverse problem the simultaneous cooling of the lesion and surrounding healthy skin is needed, as well as the data processing to obtain the temperature difference.

Measurement data in this paper have been generated numerically by solving direct bio-heat problems for Clark II and Clark IV to evaluate the exact solution of the inverse bioheat problem. Figure 3 shows the simulated temperature response or temperature difference between the tumour and surrounding skin during the rewarming period for the Clark IV test example, while Figure 4 shows the comparison between Clark II and Clark IV tumours. As can be seen, the temperature difference obtained by applying cold stress is higher than for the steady-state case, even for the early stage tumour, and therefore much easier to observe with the IR camera, which is for position $p$ and time $t$ defined as:

$$
\Delta T_{p, t}=T\left(r_{p}, H, t_{t}\right)-T\left(D / 2, H, t_{t}\right),
$$

where $r_{p}$ represent the radial position of the measurement point and $t_{t}$ the time of the measurement. The measurement data have not been taken for the whole skin surface 
but just for points above the skin tumour at $n_{p}=16$ equally spaced positions in the radial range of $r_{p} \in[0,6 \mathrm{~mm}]$ due to the $3 \mathrm{D}$-axisymmetrical solution, and at each second during the rewarming phase, which means that the number of measurements in time has been $n_{t}=600$. We found that this measurement resolution is fine enough to capture the dynamic change of temperature difference and to be able to evaluate the four tumour parameters.
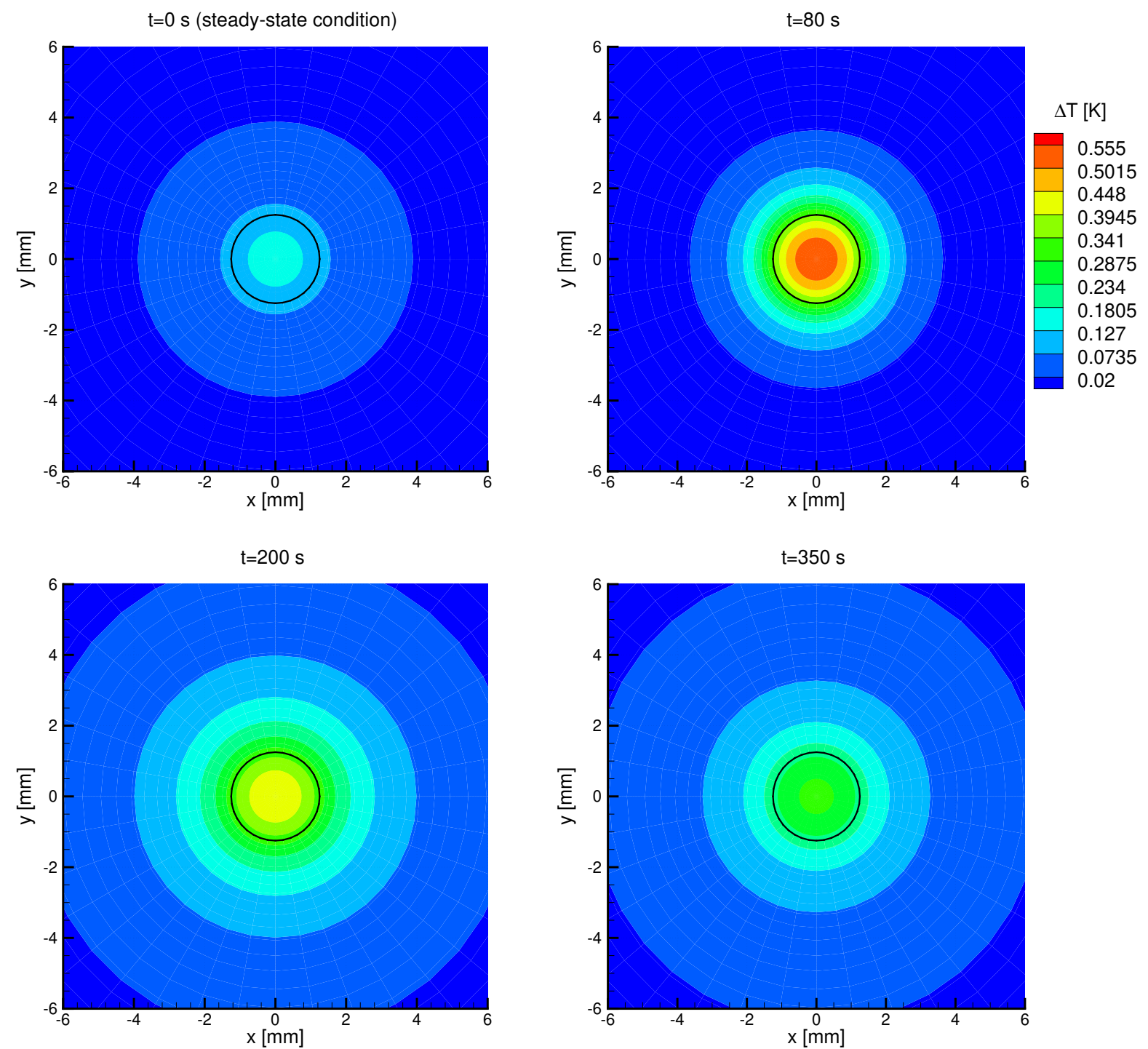

Figure 3: Contours of simulated temperature difference at skin surface for Clark IV during the dynamic thermography procedure at different times.

During the dynamic thermography IR camera captures the absolute surface temperature response of the underlying lesion and healthy skin for the recovery phase in the planar area. To use the proposed model or approach the temperature difference compared to the healthy surrounding skin in the radial direction from the lesion centre is needed, which can be from a practical point of view sometimes difficult to obtain, especially when the lesion is not symmetrical or convex, due to the non-circular isotherms. Shape of the lesion and with this positioning of the centre can present the main concern. To overcome this we can find the position of the center by finding the maximum temperature during 

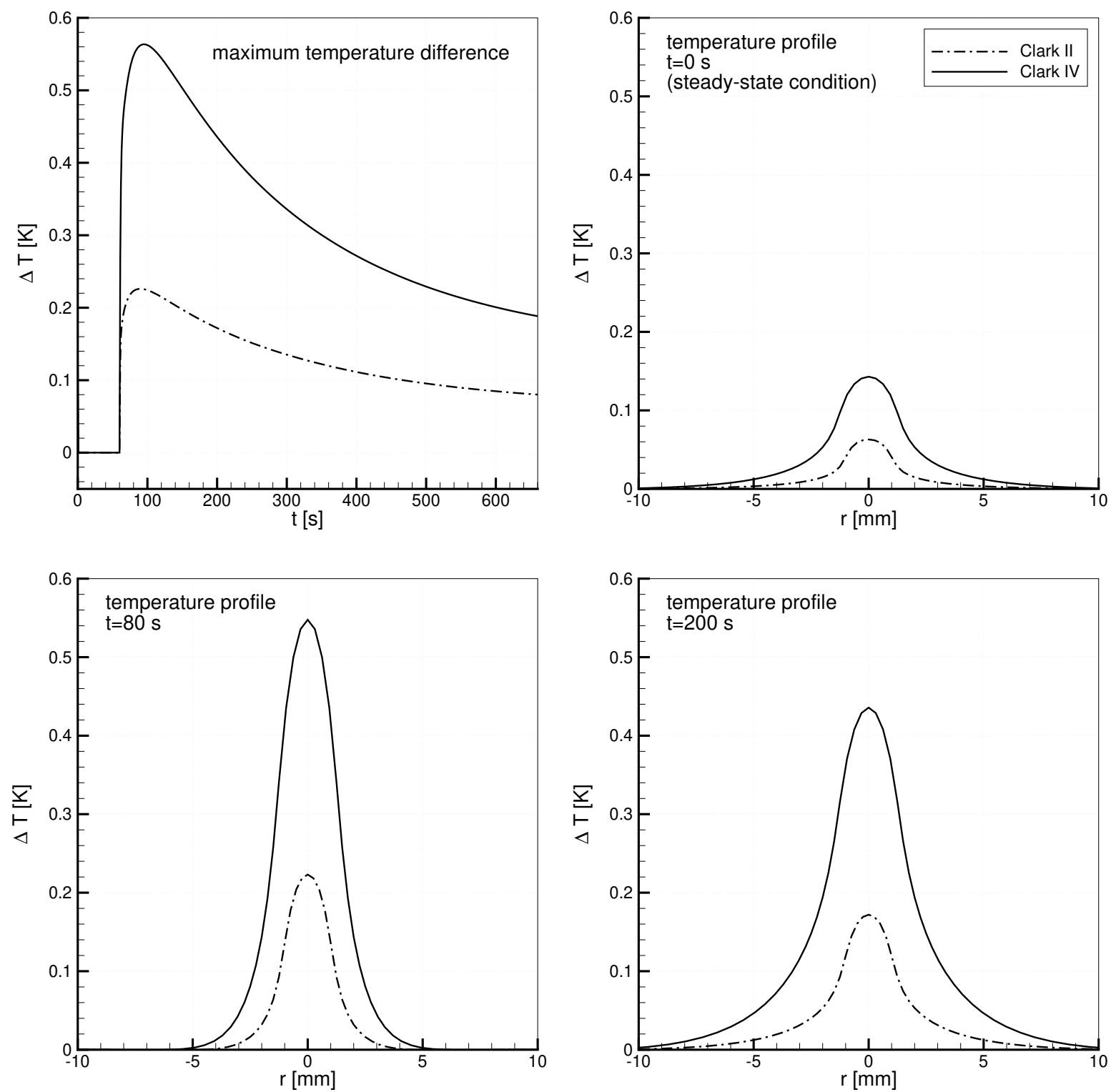

Figure 4: Response for Clark II and Clark IV tumours showing the maximum temperature difference and profile through time.

the recovery phase or the region of the maximum temperature approximated with a circle, where centre of the circle represents the lesion centre. After finding the centre the temperature measurement can be averaged by the angle to obtain the circular isotherms and the temperature difference compared to the isotherm away from the lesion. The processed data are then used in the proposed numerical model to obtain the searched tumour parameters. This way the obtained parameters for the observed irregular tumour represent not the actual values but the averaged values for the lesion approximated by the cylindrical shape that is still valuable for the diagnostic. However, if the lesion shape deviates to much from the circular one, the obtain values will not be realistic and the numerical model should be improved changing the lesion geometry to describe the observed lesion more accurately.

To mimic the real measurement data in this paper, we add white noise to the simulated 
temperature difference response as:

$$
\Delta T_{m, p, t}=\Delta T_{s, p, t}+\xi \Delta T_{e r r}
$$

where $\xi$ represents a random number; $\xi \in[-1,1]$, and $\Delta T_{\text {err }}$ a level of temperature uncertainty, while the second term on the right hand side represents the temperature deviation or noise. Different authors used different levels of uncertainty from $10 \mathrm{mK}$ up to $100 m K[15,42,48,49]$. Çetingül and Herman [39] used equipment that had an uncertainty of $25 \mathrm{mK}$, while Stra̧kowska et al. [19] used an IR camera with $20 \mathrm{mK}$, which is possible to obtain using laboratory equipment. Modern IR cameras can obtain the NETD (Noise Equivalent Temperature Difference) value of less than $30 \mathrm{mK}$. Thus, we investigate both test cases under three different levels; $0 \mathrm{mK}, 25 \mathrm{mK}$ and $50 \mathrm{mK}$. The first one represents exact measurement data, while the last two represent low and high levels of noise. In the last two cases, the measurement data do not follow the numerical model anymore. Because the noisy measurement data are generated randomly we generated five different measurement sets for each noise level, not just one. In this way, it is possible to test how the randomness of the white noise affects the inverse solution.

For a clearer presentation, Figure 5 shows the exact and noisy measurement data for Clark II and Clark IV tumours during the rewarming period, while Figure 6 shows the randomness of the generated data (different sets) for the uncertainty level of $50 \mathrm{mK}$. As can be seen from Figure 5, the level can affect the temperature response for the Clark II tumour more than for Clark IV, which makes solving inverse problems more difficult and poor accuracy to be expected for early stage tumours.

\subsection{Numerical model error}

In reality the numerical model is never an identical representation of the examined tissue, due to the uncertainty of model parameters, mathematical model, boundary conditions, geometry, etc. and therefore, does not describe the bioheat transfer exactly or realistically. In the literature we can find a large deviation of material properties, layer thicknesses $[39,61,62]$ and other model parameters, and for this reason they are usually taken at an average value or estimated. Because the solution of the inverse problem depends on the numerical model, we should also test how the uncertainty of model parameters or error in the numerical model used in this paper affects the solution.

Therefore, this paper also covers how the uncertainty of material properties for skin, tumour, fat and some boundary conditions affect the inverse solution, which is the novelty of this paper. We carried out a study in [41] on the sensitivity of the model parameters that showed a higher sensitivity for specific heat and thermal conductivity of the skin layer and tumour, tumour size, its blood perfusion rate and thermoregulation coefficient, as well as higher sensitivity for blood properties and arterial blood temperature. Because of the higher sensitivity of the tumour blood perfusion rate, its size and thermoregulation coefficient, we decided to search for these variables in this inverse analysis study. However, we would also like to test how the change of value of other model parameters affects the inverse solution, and which of them should be determined as accurately as possible in the numerical model to reflect the real experimental data and to obtain an accurate inverse solution. The chosen parameters are shown in Table 2, where we omit the analysis for the epidermis because of the relative small thickness as shown in Table 1 and low sensitivity, as well as the muscle tissue, which does not affect the tumour temperature 

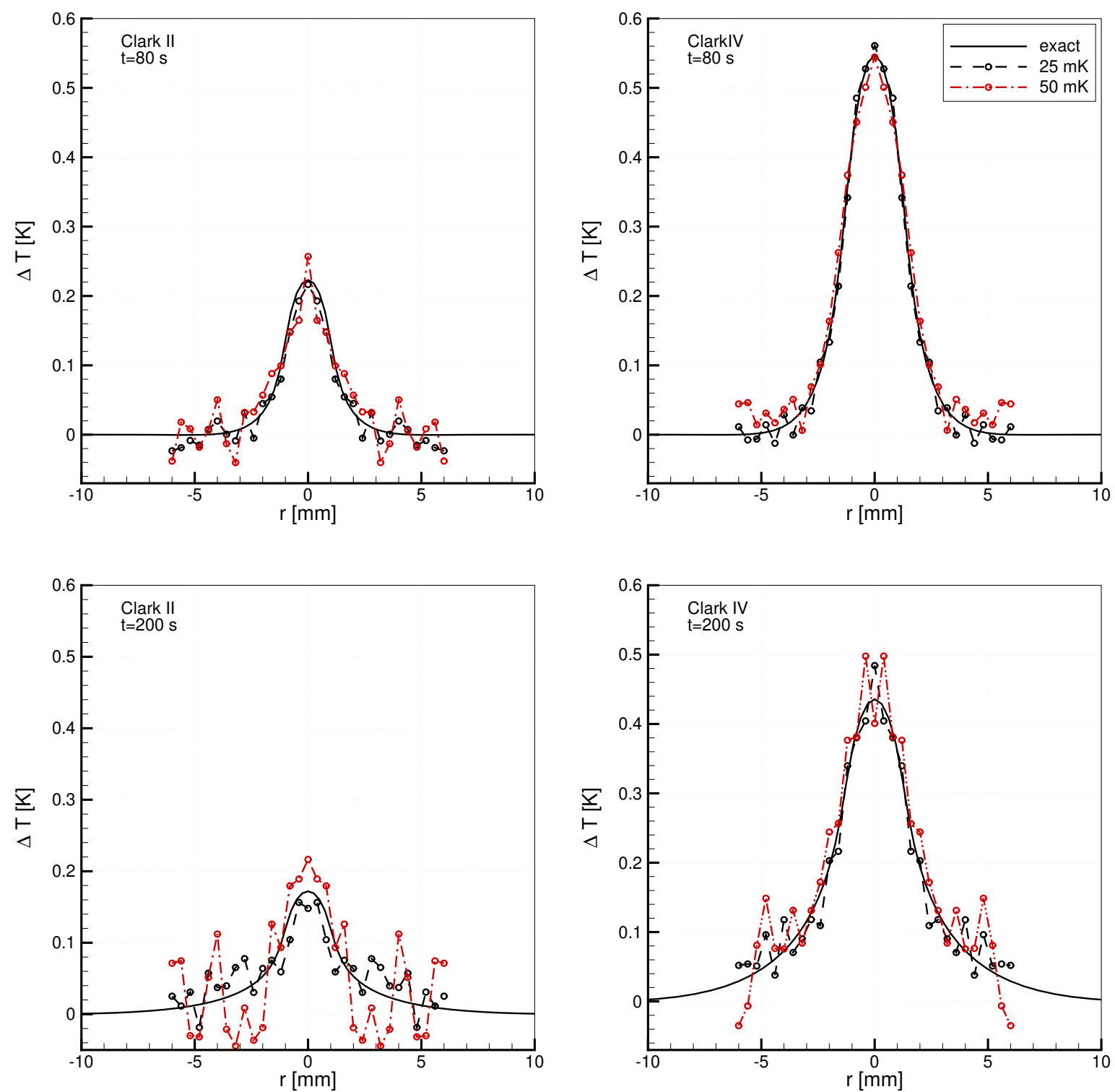

Figure 5: Exact and noisy measurement data for Clark II and Clark IV test example.

response [41]. The parameters that can be easily or accurately obtained have also been omitted in this analysis, like cooling temperature and time, density and heat capacity of the blood, etc. However, to be able to compare the inverse solution and model error, the model parameters have been changed by the same relative value; $\pm 1 \%$ and $\pm 5 \%$, while we should have in mind that uncertainty for some parameters can go up to $\pm 50 \%$.

\section{Results and discussion}

The solution of the inverse problem has been tested on numerically generated data with and without noise, prescribing unknown parameters. In this way, the exact solution of the problem is known and the analysis of the inverse problem can be verified. The exact solution for Clark II is $d=2.0 \mathrm{~mm}, h=0.44 \mathrm{~mm}, \omega_{b}=0.0063 \mathrm{~s}^{-1}$ and $Q_{10}=1.1$, while for Clark IV only the diameter and thickness are changed to $d=2.5 \mathrm{~mm}$ and $h=1.1 \mathrm{~mm}$. 

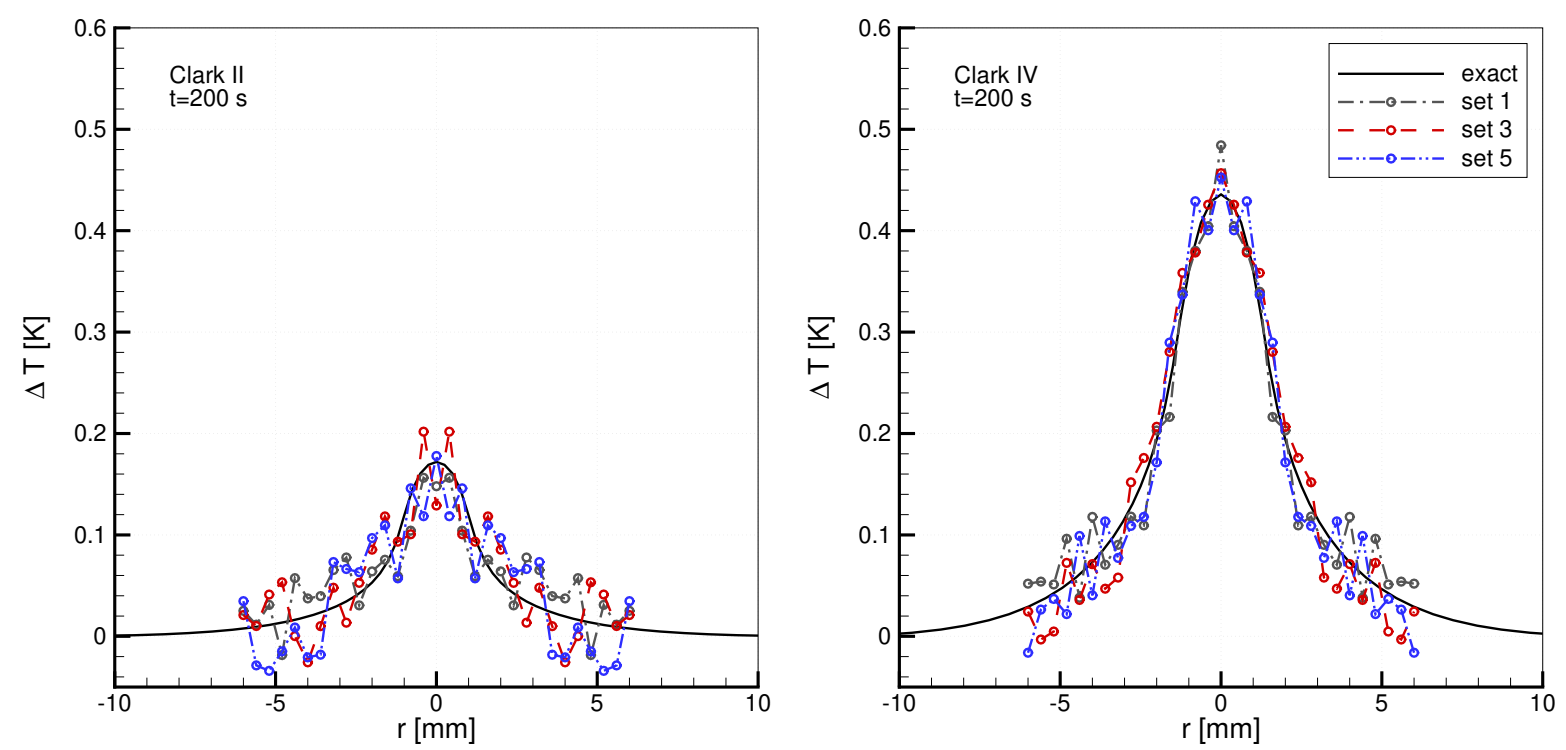

Figure 6: Comparison of different sets of $50 \mathrm{mK}$ noisy measurement data at $t=200 \mathrm{~s}$ for Clark II and Clark IV test example.

To test the optimisation algorithm, stability of results and uniqueness of the solution, we run the algorithm using five different starting points $y_{0}$. Starting points have been chosen randomly, some in close proximity to the exact solution and some far away. Starting points for Clark II and Clark IV test examples are shown in Table 3.

The results are presented using tables, which is the most appropriate to show the evaluated parameters of the inverse bioheat problem. This section covers the analysis of the starting point, measurement noise, randomness of the measurement data and model error on the inverse solution, as well as a discussion of the results. First, the results are presented for the exact model, which means that all the model parameters like specific heat, layer thickness, thermal conductivity of the tissue, etc., have been prescribed exactly the same as when the measurement data have been generated. This means that the numerical model follows the measurement data or that the model describes the examined tissue exactly. In the second part, the results for non-exact models are shown, by changing

\begin{tabular}{|c|c|c|c|c|c|c|c|}
\hline material \parameter & $\rho$ & $c_{p}$ & $k$ & $q_{m, b a s}$ & $\omega_{b, b a s}$ & $h$ & $Q_{10}$ \\
\hline epidermis & & & & & & & \\
\hline papillary dermis & & $\checkmark$ & $\checkmark$ & $\checkmark$ & $\checkmark$ & $\checkmark$ & $\checkmark$ \\
\hline reticular dermis & & $\checkmark$ & $\checkmark$ & $\checkmark$ & $\checkmark$ & $\checkmark$ & $\checkmark$ \\
\hline fat & & $\checkmark$ & $\checkmark$ & $\checkmark$ & $\checkmark$ & $\checkmark$ & $\checkmark$ \\
\hline muscle & & & & & & & \\
\hline $\begin{array}{l}\text { tumour } \\
\text { blood }\end{array}$ & & $\checkmark$ & $\checkmark$ & $\checkmark$ & & & \\
\hline bound. cond. parameter & $\alpha$ & $T_{\infty}$ & $T_{0}=T_{a}$ & $T_{c}$ & $t_{c}$ & & \\
\hline & $\checkmark$ & & $\bar{\nabla} \checkmark$ & & & & \\
\hline
\end{tabular}

Table 2: Chosen model parameters for model error analysis. 
one of the model parameters to observe how this change affects the inverse solution. The change is made for several model parameters to investigate which of them is the most crucial one and have to be determined precisely to make the estimation of the searched parameters more accurate. The model error imposes an additional error to the inverse problem, which makes parameter estimation more difficult.

\subsection{Exact model}

\subsubsection{Starting point}

The analysis of the starting point has been carried out first. This shows if the optimisation process is stable and if a unique solution is obtained. If the solution of the inverse problem changes drastically with the change of the starting point, then the problem does not have a unique solution and is therefore not solvable. Table 4 shows the results for Clark II tumour for all three levels of measurement noise using a fourth set of measurement data, together with the exact solution in bold for comparison.

As can be seen, the solution of the inverse problem does not depend on the initial starting point, which shows that the solution is unique, however there is a small but negligible variation in the inverse solution regarding the starting point when measurement noise is introduced. Similar observations have been made using different sets of measurement data, as well as for Clark IV and are therefore omitted. Regarding the solution, we can observe that under zero noise condition the exact solution can be retrieved, however an error is made with noisy measurement data. Usually the error of the estimated parameter increases with increasing noise, which depends on the randomness of the noise or generated measurement data as it will be presented in the next subsection.

Figure 7 shows the convergence of the optimisation algorithm through iteration steps for Clark II under no-noise and noisy measurement data for three different starting points, where we can observe a steady descent of the objective function for zero noise data, while for noisy data convergence is fast at the beginning and then is progressing slowly towards the end of the process, which is typical. Normally the optimisation process completes within around 15 iterations, which is fast, and the starting point does not have a huge effect on the convergence speed, especially when noise is present. The value of the

\begin{tabular}{c|c|cccc} 
Example & $y_{0}$ & $\omega_{b}[1 / \mathrm{s}]$ & $h[\mathrm{~mm}]$ & $d[\mathrm{~mm}]$ & $Q_{10}[1]$ \\
\hline \hline & 1 & 0.0080 & 0.60 & 2.30 & 1.40 \\
Clark II & 2 & 0.0060 & 0.50 & 1.90 & 1.00 \\
& 3 & 0.0050 & 0.30 & 1.70 & 1.30 \\
& 4 & 0.0040 & 0.40 & 1.20 & 1.40 \\
& 5 & 0.0060 & 0.50 & 1.50 & 1.40 \\
\hline \multirow{5}{*}{ Clark IV } & 1 & 0.0090 & 0.90 & 2.40 & 1.30 \\
& 2 & 0.0060 & 1.20 & 2.60 & 1.00 \\
& 3 & 0.0090 & 1.40 & 3.00 & 0.80 \\
& 4 & 0.0050 & 0.70 & 1.80 & 0.80 \\
& 5 & 0.0060 & 1.20 & 2.00 & 1.20
\end{tabular}

Table 3: Different starting points for optimisation process. 
objective function is of course different for zero noise and noisy measurement data, where for no noise it goes towards zero, while for the noisy data it goes towards a certain value, as can be seen in Figure 7 and Table 4. A similar behavior has been observed for Clark $\mathrm{IV}$, as well as for temperature uncertainty of $50 \mathrm{mK}$ compared to $25 \mathrm{mK}$ and is therefore omitted here.
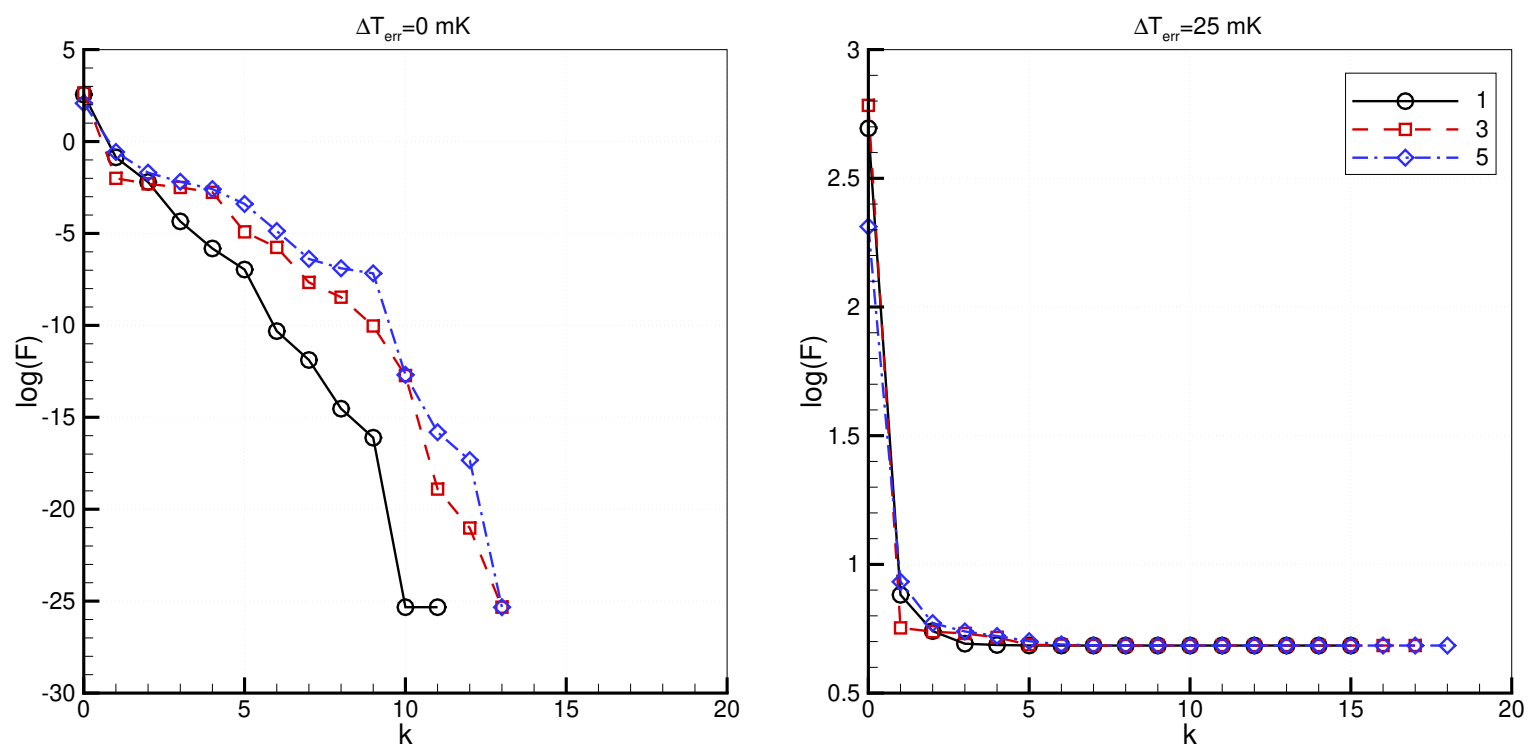

Figure 7: Convergence of LM optimisation for Clark II test example using different starting points for $0 \mathrm{mK}$ and $25 \mathrm{mK}$ measurement uncertainty.

At this point, we can conclude that the inverse solution obtained with the LM opti-

\begin{tabular}{c|c|cccc|c}
$\Delta T_{\text {err }}$ & $y_{0}$ & $\omega_{b}[1 / s]$ & $h[\mathrm{~mm}]$ & $d[\mathrm{~mm}]$ & $Q_{10}[1]$ & $F\left[K^{2}\right]$ \\
\hline \hline & exact & $\mathbf{0 . 0 0 6 3 0 0}$ & $\mathbf{0 . 4 4 0 0}$ & $\mathbf{2 . 0 0 0}$ & $\mathbf{1 . 1 0 0}$ & \\
\hline \multirow{5}{*}{$0 \mathrm{mK}$} & 1 & 0.006300 & 0.4400 & 2.000 & 1.100 & $0.163 \mathrm{E}-09$ \\
& 2 & 0.006300 & 0.4400 & 2.000 & 1.100 & $0.347 \mathrm{E}-08$ \\
& 3 & 0.006300 & 0.4400 & 2.000 & 1.100 & $0.739 \mathrm{E}-09$ \\
& 4 & 0.006300 & 0.4400 & 2.000 & 1.100 & $0.118 \mathrm{E}-07$ \\
& 5 & 0.006300 & 0.4400 & 2.000 & 1.100 & $0.297 \mathrm{E}-07$ \\
\hline \multirow{5}{*}{$5 \mathrm{mK}$} & 1 & 0.006647 & 0.4166 & 2.000 & 1.102 & $0.198 \mathrm{E}+01$ \\
& 2 & 0.006649 & 0.4165 & 2.000 & 1.102 & $0.198 \mathrm{E}+01$ \\
& 3 & 0.006650 & 0.4164 & 2.000 & 1.102 & $0.198 \mathrm{E}+01$ \\
& 4 & 0.006648 & 0.4165 & 2.000 & 1.102 & $0.198 \mathrm{E}+01$ \\
& 5 & 0.006649 & 0.4165 & 2.000 & 1.102 & $0.198 \mathrm{E}+01$ \\
\hline \multirow{5}{*}{$50 \mathrm{mK}$} & 1 & 0.006796 & 0.4064 & 1.996 & 1.102 & $0.798 \mathrm{E}+01$ \\
& 2 & 0.006795 & 0.4065 & 1.996 & 1.102 & $0.798 \mathrm{E}+01$ \\
& 3 & 0.006804 & 0.4059 & 1.996 & 1.102 & $0.798 \mathrm{E}+01$ \\
& 4 & 0.006790 & 0.4068 & 1.996 & 1.102 & $0.798 \mathrm{E}+01$ \\
& 5 & 0.006798 & 0.4063 & 1.996 & 1.102 & $0.798 \mathrm{E}+01$
\end{tabular}

Table 4: Solution of inverse problem for Clark II test example using different starting points and exact numerical model. 
misation method does not depend on the initial starting point, convergence is fast and that a unique solution of the problem is obtained. For zero noise measurement data and exact numerical model, the exact solution can be obtained, while for noisy data an error is made, as can bee seen from Table 4. However, a good approximation to the exact solution can still be obtained using low noise measurement data, while the diameter and thermoregulation coefficient could be evaluated more precisely regardless of the measurement noise.

\subsubsection{Measurement data}

As discussed in subsection 3.1, we would like to test the sensitivity of the inverse solution regarding the randomness of the noise. Therefore, five different measurement data sets have been prepared to test both inverse examples, as shown in Figure 6. Because the solution does not depend on the starting point, the starting point has been taken randomly from the set. For Clark II we choose starting point 1 while for Clark IV point 5, which have been kept fixed for all our further calculations.

\begin{tabular}{c|c|cccc|cccc|c}
$\Delta T_{\text {err }}$ & Set & $\omega_{b}[1 / s]$ & $h[\mathrm{~mm}]$ & $d[\mathrm{~mm}]$ & $Q_{10}[1]$ & $\omega_{b}[\%]$ & $h[\%]$ & $d[\%]$ & $Q_{10}[\%]$ & $F\left[K^{2}\right]$ \\
\hline \hline & exact & $\mathbf{0 . 0 0 6 3 0 0}$ & $\mathbf{0 . 4 4 0 0}$ & $\mathbf{2 . 0 0 0}$ & $\mathbf{1 . 1 0 0}$ & & & & & \\
\hline \multirow{5}{*}{$25 \mathrm{mK}$} & 1 & 0.005670 & 0.5058 & 1.974 & 1.098 & 9.99 & 14.95 & 1.31 & 0.20 & $0.204 \mathrm{E}+01$ \\
& 2 & 0.006296 & 0.4310 & 2.027 & 1.100 & 0.06 & 2.05 & 1.33 & 0.01 & $0.199 \mathrm{E}+01$ \\
& 3 & 0.005845 & 0.4867 & 1.970 & 1.094 & 7.23 & 10.61 & 1.51 & 0.50 & $0.203 \mathrm{E}+01$ \\
& 4 & 0.006647 & 0.4166 & 2.000 & 1.102 & 5.51 & 5.32 & 0.02 & 0.19 & $0.198 \mathrm{E}+01$ \\
& 5 & 0.005323 & 0.5317 & 1.985 & 1.089 & 15.51 & 20.84 & 0.73 & 1.04 & $0.201 \mathrm{E}+01$ \\
\hline \multirow{5}{*}{$50 \mathrm{mK}$} & 1 & 0.006396 & 0.4394 & 1.999 & 1.115 & 1.52 & 0.13 & 0.03 & 1.32 & $0.808 \mathrm{E}+01$ \\
& 2 & 0.005399 & 0.5432 & 1.946 & 1.093 & 14.34 & 23.46 & 2.72 & 0.60 & $0.801 \mathrm{E}+01$ \\
& 3 & 0.008347 & 0.3321 & 1.999 & 1.128 & 32.50 & 24.53 & 0.03 & 2.54 & $0.803 \mathrm{E}+01$ \\
& 4 & 0.006796 & 0.4064 & 1.996 & 1.102 & 7.88 & 7.64 & 0.20 & 0.19 & $0.798 \mathrm{E}+01$ \\
& 5 & 0.006987 & 0.3854 & 2.016 & 1.101 & 10.91 & 12.41 & 0.82 & 0.11 & $0.795 \mathrm{E}+01$
\end{tabular}

Table 5: Solution of inverse problem and relative error for Clark II test example using different sets of measurement data and exact numerical model.

Table 5 shows the results for the Clark II test using different sets of measurement data for temperature uncertainty of $25 \mathrm{mK}$ and $50 \mathrm{mK}$, together with the relative error compared to the exact solution. As can be observed, the randomness of the noise is affecting the inverse solution and for certain data sets the error can be quite high, especially for the estimation of blood perfusion and thickness of the tumour.

Convergence of the LM optimisation algorithm for different sets of noisy measuring data is displayed in Figure 8, which shows fast convergence at the first five steps and slow at the end, as observed when testing different starting points. The number of iteration steps used to find the solution was around 15 . There is a slight difference between different measurement sets, achieving lower value of the objective function, as can be seen from Table 5. Similar observation has also been made for the Clark IV test and is therefore omitted here. However, the LM method proved to be stable and appropriate to solve the inverse bioheat problem. 

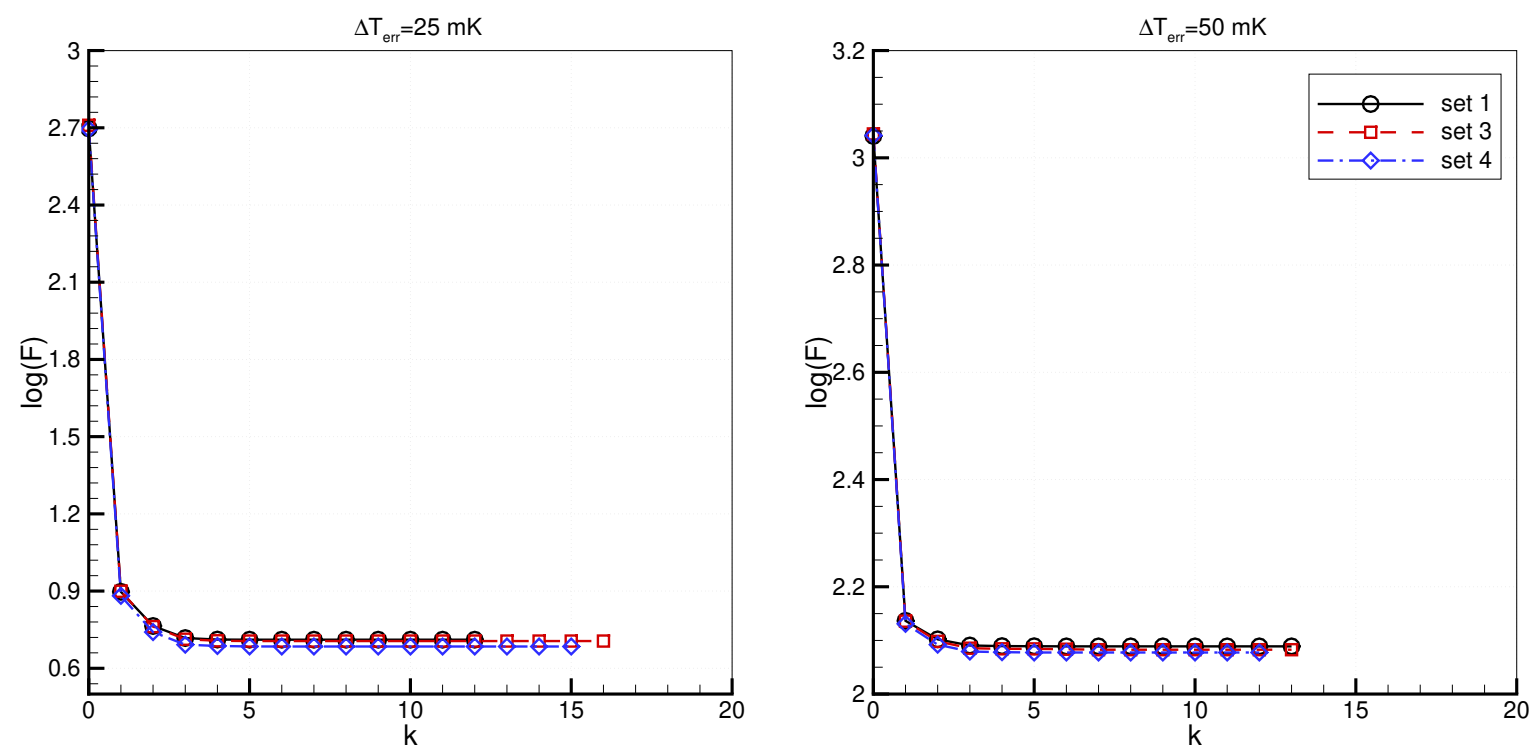

Figure 8: Convergence of LM optimisation for Clark II test example using different measurement data set for $25 \mathrm{mK}$ and $50 \mathrm{mK}$ measurement uncertainty.

From this analysis, we can conclude that the solution of the inverse problem depends on the level and randomness of the noise. Therefore, when testing the inverse problem numerically, it is needed to test the problem on different randomly generated measurement data. In this paper, we generated five different sets for each noise level and we statistically analyse the results from each set to make a conclusion on how the noise level affects the inverse solution as shown in the next subsection.

\subsubsection{Solution for Clark II and Clark IV}

As seen from Table 5, the inverse analysis solution depends on the measurement data set, where in some cases the error for some parameters can be quite large. To be able to discuss the inverse solution and how it is affected by measurement noise, we used a statistical approach to analyse the solution. We are aware that five different solutions is a small sample size, which makes statistical analysis not accurate, however it still gives an insight on how the measurement noise affects the inverse solution.

Because of the variation of evaluated parameters for each measurement set the average value and standard deviation of each parameter has been calculated, together with the

\begin{tabular}{|c|c|c|c|c|c|c|c|c|}
\hline$\Delta T_{e r r}$ & $\overline{\omega_{b}} \pm \sigma_{\omega}[1 / s]$ & $\bar{h} \pm \sigma_{h}[\mathrm{~mm}]$ & $\bar{d} \pm \sigma_{d}[\mathrm{~mm}]$ & $\overline{Q_{10}} \pm \sigma_{Q}[1]$ & $\overline{\omega_{b}}[\%]$ & $\bar{h}[\%]$ & $\bar{d}[\%]$ & $\overline{Q_{10}}[\%]$ \\
\hline exact & 0.006300 & 0.4400 & 2.000 & $\overline{1.100}$ & & & & \\
\hline $25 \mathrm{mK}$ & $\begin{array}{c}0.005956 \pm \\
0.000522\end{array}$ & $\begin{array}{c}0.4743 \pm \\
0.0491\end{array}$ & $\begin{array}{c}1.991 \pm \\
0.023\end{array}$ & $\begin{array}{c}1.097 \pm \\
0.005\end{array}$ & 7.66 & 10.75 & 0.98 & 0.39 \\
\hline $50 \mathrm{mK}$ & $\begin{array}{c}0.006785 \pm \\
0.001067\end{array}$ & $\begin{array}{c}0.4213 \pm \\
0.0785\end{array}$ & $\begin{array}{c}1.991 \pm \\
0.027\end{array}$ & $\begin{array}{c}1.108 \pm \\
0.014\end{array}$ & 13.42 & 13.63 & 0.76 & 0.95 \\
\hline
\end{tabular}

Table 6: Solution of inverse problem for Clark II test example showing the average value of evaluated parameters, standard deviation and average error. 
average error. Therefore, Tables 6 and 7 show the results for Clark II and Clark IV, respectively, using only noisy measurement data. In the previous subsection, we can see from Table 4 that an exact estimation of parameters is obtained using zero noise data, regardless of starting point, however this is only interesting from the numerical point of view and is not feasible in practical problems. As can be observed from Table 6 for Clark II, the parameters can be estimated with relatively good accuracy for low noise level, while for high level the evaluation of blood perfusion rate and thickness of the tumour exceeds $10 \%$ error. However, the diameter and thermoregulation coefficient are estimated within $1 \%$ regardless of the noise. We can also observe that the standard deviation of each parameter is increased with noise, which is reasonable. However, for Clark IV, the estimation of all four parameters is much more accurate than for Clark II as shown in Table 7. The error for low level of noise is below $3 \%$ and similar to the Clark II test example, and the error for diameter and thermoregulation coefficient of the tumour is very low. Also, the standard deviation of parameters is smaller for Clark IV than for Clark II, showing that the measurement noise does not influence the thermal signature of the later stage tumour as much as for early stage, as can be seen from Figures 5 or 6 . Here, we should mention that the error of blood perfusion rate and thickness of the tumour are connected, because these two parameters are interdependent. If the blood perfusion rate is evaluated higher than the exact one, then the thickness will be underestimated and vice versa, which can be observed from Tables 6 and 7 .

We can conclude that all four parameters can be estimated, especially the diameter and thermoregulation coefficient, regardless of the noise and tumour stage. While the estimation error for blood perfusion rate and thickness is low for later tumour stage, the parameters can still be evaluated for skin tumour at an early stage for low measurement noise.

\subsection{Model error}

The uncertainty of material properties, mathematical model or boundary conditions of the numerical model can influence the accuracy of the inverse solution. Therefore, parameters shown in Table 2 have been chosen to analyse the inverse solution sensitivity to $\pm 1 \%$ and $\pm 5 \%$ relative change, where parameters for papillary dermis and reticular dermis (skin) have been changed simultaneously due to having the same value as can be seen in Table 1.

Table 8 shows the solutions for uncertainty of skin heat capacity under exact mea-

\begin{tabular}{|c|c|c|c|c|c|c|c|c|}
\hline$\Delta T_{e r r}$ & $\overline{\omega_{b}} \pm \sigma_{\omega}[1 / s]$ & $\bar{h} \pm \sigma_{h}[\mathrm{~mm}]$ & $\bar{d} \pm \sigma_{d}[m m]$ & $\overline{Q_{10}} \pm \sigma_{Q}[1]$ & $\overline{\omega_{b}}[\%]$ & $\bar{h}[\%]$ & $\bar{d}[\%]$ & $\overline{Q_{10}}[\%]$ \\
\hline exact & 0.006300 & 1.100 & 2.500 & 1.100 & & & & \\
\hline $25 \mathrm{mK}$ & $\begin{array}{c}0.006260 \pm \\
0.000117\end{array}$ & $\begin{array}{c}1.1135 \pm \\
0.0364\end{array}$ & $\begin{array}{c}2.498 \pm \\
0.010\end{array}$ & $\begin{array}{c}1.102 \pm \\
0.002\end{array}$ & 1.46 & 2.63 & 0.29 & 0.21 \\
\hline $50 \mathrm{mK}$ & $\begin{array}{c}0.006326 \pm \\
0.000274\end{array}$ & $\begin{array}{c}1.1046 \pm \\
0.1025\end{array}$ & $\begin{array}{c}2.499 \pm \\
0.038\end{array}$ & $\begin{array}{c}1.102 \pm \\
0.010\end{array}$ & 3.05 & 7.20 & 1.25 & 0.74 \\
\hline
\end{tabular}

Table 7: Solution of inverse problem for Clark IV test example showing the average value of evaluated parameters, standard deviation and average error. 
surement data for Clark II and Clark IV tumours, where the solution error increases with the specific heat change. If the skin heat capacity is underestimated, the estimation of blood perfusion rate, tumour diameter and thermoregulation coefficient will be also underestimated, while thickness will be overestimated due to the lower estimated blood perfusion rate, and vice versa. As already described, the blood perfusion rate and tumour thickness are interdependent and also the error for these two parameters will be connected and can be looked as one. The $5 \%$ change of skin heat capacity will affect the estimation of tumour thickness and blood perfusion rate the most, where the error will be around $10 \%$ for thickness and $6 \%$ for blood perfusion rate for the Clark II example. The uncertainty will also affect the estimation of the thermoregulation coefficient, while the diameter will still be determined accurately. For Clark IV, the uncertainty in skin heat capacity affects the blood perfusion rate the most, while other parameters can be determined accurately with an error lower than $2 \%$. From the results shown in Table 8 , we can conclude that uncertainty of skin heat capacity will have the largest effect on the estimation of blood perfusion rate and tumour thickness and for accurate estimation of these parameters, heat capacity of the skin should be determined in the numerical model as accurately as possible, especially when the estimation of searched parameters is needed for an early stage tumour.

From Table 8, we analyse the effect of skin specific heat uncertainty on inverse solution under zero noise measurement data, while Table 9 shows the effect of measurement noise, showing only the average estimation error. The average error of the estimated parameters is even larger under noisy measurement, especially for Clark II. The error for blood perfusion rate and tumour thickness becomes higher than $13 \%$ for $50 \mathrm{mK}$ of measurement uncertainty, regardless of the level of skin specific heat change, which means that measurement noise in Clark II is prevailing, and similar conclusion can be made for temperature uncertainty of $25 \mathrm{mK}$. However, for Clark IV the parameter estimation error does not change so significantly and the inverse solution is still controlled by model error or uncertainty.

The error of the inverse solution under noisy measurement data and model error can be roughly estimated as the sum of separated errors made by the measurement noise under an exact model and error for model uncertainty under zero noise measurement data.

\begin{tabular}{c|c|cccc|cccc} 
Example & $c_{p}[\mathrm{~J} / \mathrm{kgK}]$ & $\omega_{b}[1 / \mathrm{s}]$ & $h[\mathrm{~mm}]$ & $d[\mathrm{~mm}]$ & $Q_{10}[1]$ & $\overline{\omega_{b}}[\%]$ & $\bar{h}[\%]$ & $\bar{d}[\%]$ & $\overline{Q_{10}}[\%]$ \\
\hline \hline exact & & $\mathbf{0 . 0 0 6 3 0 0}$ & $\mathbf{1 . 1 0 0}$ & $\mathbf{2 . 5 0 0}$ & $\mathbf{1 . 1 0 0}$ & & & & \\
\hline \multirow{3}{*}{ Clark II } & $3135.0(-5 \%)$ & 0.005979 & 0.4836 & 1.980 & 1.062 & 5.09 & 9.90 & 0.98 & 3.50 \\
& $3267.0(-1 \%)$ & 0.006223 & 0.4494 & 1.996 & 1.092 & 1.23 & 2.14 & 0.21 & 0.72 \\
& $3333.0(+1 \%)$ & 0.006377 & 0.4311 & 2.004 & 1.107 & 1.22 & 2.02 & 0.20 & 0.67 \\
& $3465.0(+5 \%)$ & 0.006738 & 0.3948 & 2.020 & 1.135 & 6.95 & 10.27 & 1.00 & 3.21 \\
\hline \multirow{3}{*}{ Clark IV } & $3135.0(-5 \%)$ & 0.005999 & 1.1183 & 2.506 & 1.121 & 4.78 & 1.66 & 0.26 & 1.91 \\
& $3267.0(-1 \%)$ & 0.006236 & 1.1048 & 2.501 & 1.104 & 1.02 & 0.44 & 0.04 & 0.38 \\
& $3333.0(+1 \%)$ & 0.006366 & 1.0948 & 2.499 & 1.096 & 1.05 & 0.47 & 0.03 & 0.38 \\
& $3465.0(+5 \%)$ & 0.006643 & 1.0713 & 2.497 & 1.079 & 5.44 & 2.61 & 0.13 & 1.91
\end{tabular}

Table 8: Inverse solution for Clark II and Clark IV test example under skin specific heat uncertainty. 
Therefore, to present more clearly the effect of model uncertainty, only the estimated parameter error for zero noise measurement data will be shown. Tables 10 and 11 show the average parameter error estimation for $1 \%$ and $5 \%$ uncertainty, respectively. As can be observed, the metabolic heat generation does not affect the inverse solution, regardless of the tumour stage, as well as blood perfusion rate and thermoregulation coefficient due to low sensitivity [41]. The error is below $0.5 \%$ for $5 \%$ while the highest effect among

\begin{tabular}{c|c|cccc|cccc}
\multicolumn{2}{l|}{} & \multicolumn{5}{|c|}{ Clark II } & \multicolumn{4}{c}{ Clark IV } \\
\hline$\Delta T_{\text {err }}$ & $c_{p}[\%]$ & $\overline{\omega_{b}}[\%]$ & $\bar{h}[\%]$ & $\bar{d}[\%]$ & $\overline{Q_{10}}[\%]$ & $\overline{\omega_{b}}[\%]$ & $\bar{h}[\%]$ & $\bar{d}[\%]$ & $\overline{Q_{10}}[\%]$ \\
\hline \hline \multirow{3}{*}{$25 \mathrm{mK}$} & $-5 \%$ & 9.61 & 17.26 & 1.56 & 3.87 & 5.79 & 4.82 & 0.44 & 2.14 \\
& $-1 \%$ & 8.17 & 11.31 & 1.10 & 1.06 & 2.08 & 2.97 & 0.30 & 0.53 \\
& $+1 \%$ & 7.57 & 10.23 & 0.93 & 0.50 & 1.31 & 2.45 & 0.28 & 0.26 \\
& $+5 \%$ & 7.11 & 8.16 & 0.85 & 3.04 & 4.93 & 2.39 & 0.27 & 1.81 \\
\hline \multirow{3}{*}{$50 \mathrm{mK}$} & $-5 \%$ & 10.83 & 13.19 & 1.40 & 2.62 & 4.79 & 7.40 & 1.27 & 2.05 \\
& $-1 \%$ & 12.43 & 13.37 & 0.88 & 1.00 & 2.73 & 6.12 & 1.06 & 0.78 \\
& $+1 \%$ & 14.41 & 14.64 & 0.77 & 1.35 & 2.78 & 5.67 & 1.02 & 0.63 \\
& $+5 \%$ & 19.02 & 18.78 & 1.22 & 3.75 & 6.22 & 5.20 & 0.95 & 0.77
\end{tabular}

Table 9: Average error of the inverse solution for Clark II and Clark IV test example for skin specific heat uncertainty under noisy measurement data.

\begin{tabular}{c|c|cccc|cccc}
\multicolumn{1}{c|}{} & \multicolumn{5}{|c|}{ Clark II } & \multicolumn{4}{c}{ Clark IV } \\
\hline \multirow{2}{*}{ Parameter } & Material & $\overline{\omega_{b}}[\%]$ & $\bar{h}[\%]$ & $\bar{d}[\%]$ & $\overline{Q_{10}}[\%]$ & $\overline{\omega_{b}}[\%]$ & $\bar{h}[\%]$ & $\bar{d}[\%]$ & $\overline{Q_{10}}[\%]$ \\
\hline \multirow{3}{*}{$c_{p}$} & skin & 1.22 & 2.08 & 0.20 & 0.70 & 1.03 & 0.45 & 0.04 & 0.38 \\
& fat & 0.20 & 0.48 & 0.04 & 0.13 & 0.15 & 0.19 & 0.05 & 0.20 \\
& tumour & 2.20 & 2.30 & 0.19 & 1.15 & 0.04 & 0.09 & 0.05 & 0.85 \\
\hline \multirow{3}{*}{$\lambda$} & skin & 1.58 & 0.85 & 0.43 & 0.79 & 3.95 & 3.98 & 0.09 & 0.41 \\
& fat & 0.23 & 0.14 & 0.11 & 0.19 & 0.00 & 0.85 & 0.03 & 0.27 \\
& tumour & 0.20 & 1.30 & 0.21 & 0.49 & 1.50 & 1.65 & 0.09 & 0.30 \\
\hline \multirow{3}{*}{$q_{m, \text { bas }}$} & skin & 0.00 & 0.01 & 0.00 & 0.00 & 0.00 & 0.00 & 0.00 & 0.00 \\
& fat & 0.00 & 0.00 & 0.00 & 0.00 & 0.00 & 0.00 & 0.00 & 0.00 \\
\multirow{2}{*}{$\omega_{m, \text { bas }}$} & tumour & 0.00 & 0.04 & 0.00 & 0.01 & 0.02 & 0.05 & 0.01 & 0.02 \\
\hline \multirow{2}{*}{$Q_{10}$} & skin & 0.00 & 0.11 & 0.00 & 0.04 & 0.00 & 0.00 & 0.00 & 0.00 \\
& fat & 0.00 & 0.01 & 0.00 & 0.00 & 0.02 & 0.05 & 0.01 & 0.01 \\
\hline \multirow{2}{*}{$h$} & skin & 0.05 & 0.02 & 0.01 & 0.03 & 0.08 & 0.09 & 0.01 & 0.02 \\
& fat & 0.00 & 0.00 & 0.00 & 0.00 & 0.02 & 0.05 & 0.01 & 0.01 \\
\hline \hline \multirow{2}{*}{ Bound. cond. } & pap. dermis & 0.06 & 0.15 & 0.04 & 0.12 & 0.16 & 0.21 & 0.04 & 0.19 \\
& ret. dermis & 0.10 & 0.09 & 0.05 & 0.11 & 0.09 & 0.12 & 0.05 & 0.14 \\
& fat & 0.24 & 0.28 & 0.01 & 0.26 & 0.28 & 0.52 & 0.01 & 0.35 \\
\hline & Ta & 2.74 & 0.19 & 0.26 & 0.49 & 3.40 & 1.56 & 0.07 & 0.24 \\
& $\alpha$ & 0.04 & 0.57 & 0.10 & 0.23 & 0.50 & 0.20 & 0.04 & 0.22
\end{tabular}

Table 10: Average error of the inverse solution for Clark II and Clark IV test example for $1 \%$ change of model parameters under zero noise measurement data. 


\begin{tabular}{c|c|cccc|cccc}
\multicolumn{2}{c|}{} & \multicolumn{6}{|c|}{ Clark II } & \multicolumn{4}{c}{ Clark IV } \\
\hline \multirow{2}{*}{ Parameter } & Material & $\overline{\omega_{b}}[\%]$ & $\bar{h}[\%]$ & $\bar{d}[\%]$ & $\overline{Q_{10}}[\%]$ & $\overline{\omega_{b}}[\%]$ & $\bar{h}[\%]$ & $\bar{d}[\%]$ & $\overline{Q_{10}}[\%]$ \\
\hline \multirow{3}{*}{$c_{p}$} & skin & 6.02 & 10.08 & 0.99 & 3.35 & 5.11 & 2.14 & 0.19 & 1.91 \\
& fat & 1.01 & 2.37 & 0.22 & 0.67 & 0.77 & 0.94 & 0.24 & 0.99 \\
& tumour & 11.29 & 11.62 & 0.96 & 5.72 & 0.23 & 0.12 & 0.27 & 4.28 \\
\hline \multirow{3}{*}{$\lambda$} & skin & 8.81 & 8.86 & 1.89 & 3.82 & 20.86 & 24.91 & 1.05 & 1.70 \\
& fat & 1.15 & 0.72 & 0.54 & 0.93 & 0.09 & 4.23 & 0.14 & 1.36 \\
& tumour & 3.95 & 5.22 & 0.95 & 2.42 & 7.80 & 9.06 & 0.63 & 1.44 \\
\hline \multirow{3}{*}{$q_{m, \text { bas }}$} & skin & 0.01 & 0.03 & 0.00 & 0.01 & 0.02 & 0.05 & 0.01 & 0.01 \\
& fat & 0.00 & 0.01 & 0.00 & 0.00 & 0.01 & 0.00 & 0.00 & 0.00 \\
\multirow{2}{*}{$\omega_{m, \text { bas }}$} & tumour & 0.00 & 0.19 & 0.01 & 0.07 & 0.18 & 0.09 & 0.01 & 0.08 \\
\hline \multirow{2}{*}{$Q_{10}$} & skin & 0.00 & 0.55 & 0.02 & 0.22 & 0.00 & 0.00 & 0.00 & 0.00 \\
& fat & 0.01 & 0.06 & 0.00 & 0.02 & 0.05 & 0.09 & 0.01 & 0.02 \\
\hline \multirow{3}{*}{$h$} & skin & 0.24 & 0.10 & 0.03 & 0.12 & 0.17 & 0.46 & 0.01 & 0.12 \\
& fat & 0.00 & 0.02 & 0.00 & 0.01 & 0.05 & 0.09 & 0.01 & 0.02 \\
\hline \multirow{2}{*}{ Bound. cond. } & pap. dermis & 0.32 & 0.76 & 0.20 & 0.60 & 0.80 & 1.04 & 0.22 & 0.94 \\
& ret. dermis & 0.50 & 0.45 & 0.24 & 0.55 & 0.46 & 0.60 & 0.23 & 0.69 \\
& fat & 1.19 & 1.41 & 0.04 & 1.30 & 1.39 & 2.58 & 0.04 & 1.73 \\
\hline & $T_{a}$ & 14.10 & 1.06 & 1.28 & 2.48 & 17.23 & 8.01 & 0.28 & 1.21 \\
& $\alpha$ & 0.19 & 2.87 & 0.49 & 1.13 & 2.49 & 0.96 & 0.20 & 1.08
\end{tabular}

Table 11: Average error of the inverse solution for Clark II and Clark IV test example for $5 \%$ change of model parameters under zero noise measurement data.

these parameters will be on the skin blood perfusion rate and, consequently, also the skin thermoregulation coefficient $Q_{10}$. Small errors can also be expected for skin and fat thickness, where fat has the strongest effect [41]. The solution error in this case is in the same range of the blood perfusion rate, tumour thickness and thermoregulation coefficient regardless of tumour size, while usually the highest error has been made for blood perfusion rate and tumour thickness for early stage tumours as already described. However, the error made because of the thickness is small compared to the error in skin and tumour heat capacity and thermal conductivity. Model sensitivity of the tumour and skin thermal conductivity is especially visible for the Clark IV example, which is $2-2.5$ times higher than for Clark II, while for the Clark II example, tumour heat capacity has the strongest effect. The $5 \%$ error in these model parameters will produce solution errors in the range of $5-25 \%$ and should, therefore, be determined as accurately as possible to reduce the estimation error as shown in Table 10. Regarding boundary conditions, the heat transfer coefficient $\alpha$ does not have a high impact on the inverse solution, however, a large error can be made if the estimation error is high. Uncertainty in the arterial blood temperature has a much stronger effect on the inverse solution than the heat transfer coefficient, especially on blood perfusion rate, however the relative error made by the guess of $T_{a}=37^{\circ} \mathrm{C}$ is below $1 \%$, which means that solution error will be small.

To conclude, model error in fat thickness and material properties, as well as metabolic heat generation, blood perfusion rate, thermoregulation coefficient and layer thickness for skin does not drastically affect the inverse solution, and the solution error will be mostly 
controlled by measurement noise. However, specific heat and thermal conductivity of skin and tumour will have to be evaluated more precisely to estimate four tumour parameters successfully. Some of these parameters affect the inverse solution more for later stage tumour than earlier ones. The reason for this behavior can be found in tumour size, possibility to accumulate cold and rewarming speed, as well as the cold penetration depth around the lesion, which shifts the thermal response during the rewarming period and influences the accuracy of parameter estimation.

\section{Conclusion}

This paper covers the solution of inverse bioheat problems of simultaneous estimation of four different skin tumour parameters based on surface temperature differences between the lesion and health skin during the rewarming period of dynamic thermography. These four parameters are tumour thickness, diameter, blood perfusion rate and thermoregulation coefficient, which are important for diagnostic to estimate stage and invasiveness of the skin tumour. The problem in this paper is solved numerically and presents the base for further developments of non-invasive diagnostic techniques using IRT imaging.

The estimation of all four parameters is carried out on a more realistic non-homogeneous 3D numerical skin model containing a skin tumour that includes the thermoregulation response of the skin, lesion and surrounding tissue, which is important to simulate dynamic heat transfer during the cooling/rewarming process of dynamic thermography as accurately as possible, and the surface temperature response of the tumour during the rewarming period. The inverse problem is solved using an optimisation approach with deterministic LM method, which proved to be efficient for this inverse problem. The optimisation has been made based on the objective function that compared the simulated surface temperature difference between the healthy skin and lesion with the measurement data. The temperature difference between the skin and lesion reduces the error that can be made by adopting a non-exact numerical model and the difficulties in measuring the absolute temperature. In this paper, measurement data have been generated numerically for Clark II and Clark IV examples. To mimic the measurement error a $25 \mathrm{mK}$ and $50 \mathrm{mK}$ white noise has been added, based on the accuracy of modern IR cameras. Because the measurement data have been generated numerically with predetermined searched values, which were taken as exact, it has been possible to evaluate the error of the inverse solution and the success of dynamic thermography for early skin tumour detection.

The paper covers the analysis of measurement noise and model uncertainty on the inverse solution that presents a novelty in this field, especially as the additional thermoregulation coefficient can be estimated accurately under different levels of noise and tumour stage.

The optimisation algorithm and uniqueness of the inverse solution have been tested using different starting points. The solution does not depend on the starting point, and the exact solution has been obtained using zero noise measurement data and the exact model. The starting point analysis also shows the robustness of the optimisation LM algorithm, which converged in around 15 iterations steps. However, the randomness of measurement noise has a high effect on the solution. Therefore, the solution has been tested and statistically analysed for five different measurement sets to establish how the noise and randomness affect the accuracy of the inverse solution. The accuracy is affected 
by the level of noise and stage of tumour and is better for low noise level and later tumour stages, however, diameter and thermoregulation coefficient could also be determined for early stage tumours and high levels of noise. The accuracy of the blood perfusion rate and tumour thickness is most affected by noise and are correlated because of interdependency. A higher blood perfusion rate leads to thinner tumour estimation and vice versa.

The paper also covers the error analysis induced by the numerical model error. Several model parameters have been varied by $\pm 1 \%$ and $\pm 5 \%$ to evaluate which of them affects the inverse solution the most. As shown, the most important parameters are thermal conductivity and specific heat of skin and tumour, as well as arterial temperature, while metabolic heat generation, blood perfusion rate, thermoregulation coefficient and thickness of the skin and fat, together with thermal conductivity and heat capacity of the fat layer, are not so important. Because the uncertainty of arterial temperature is smaller than $1 \%$, it does not impose a problem, and therefore only the heat capacity and thermal conductivity need to be determined precisely in the numerical model to evaluate skin tumour parameters as accurately as possible, while keeping the noise level low.

As shown in the paper, dynamic thermography is a promising non-invasive approach to detect and evaluate stage and invasiveness of the skin tumour, which can improve the survival rate if detected in its early stage, as well as to evaluate some of the parameters that are not well researched as thermoregulation coefficient or response of the tumour to cold stress. Lesion parameters are evaluated based on the numerical model using cylindrical shape of the lesion, however it can be also used for the non-symmetrical lesions that can be approximated by the cylinder, for which the measurement data has to be averaged by the angle regards to the lesion centre. The accuracy of the evaluated parameters in this case depends on the shape deviation from the cylindrical one, which will be the focus of our future work.

\section{References}

[1] B. Lahiri, S. Bagavathiappan, T. Jayakumar, J. Philip, Medical applications of infrared thermography: A review, Infrared Physics \& Technology 55 (4) (2012) 221235.

[2] C. Magalhaes, R. Vardasca, J. Mendes, Recent use of medical infrared thermography in skin neoplasms, Skin Research and Technology (1-3) (2018) 1-5.

[3] G. Shi, F. Han, C. Liang, L. Wang, K. Li, A novel method of thermal tomography tumor diagnosis and its clinical practice, Applied Thermal Engineering 73 (1) (2014) 408-415.

[4] J. F. Head, F. Wang, C. A. Lipari, R. L. Elliott, The important role of infrared imaging in breast cancer, IEEE Engineering in Medicine and Biology Magazine 19 (3) (2000) 52-57.

[5] A. Di Carlo, Thermography and the possibilities for its applications in clinical and experimental dermatology, Clinics in Dermatology 13 (4) (1995) 329-336.

[6] T. M. Buzug, S. Schumann, L. Pfaffmann, U. Reinhold, J. Ruhlmann, Functional infrared imaging for skin-cancer screening, in: Engineering in Medicine and Biology 
Society, 2006. EMBS'06. 28th Annual International Conference of the IEEE, IEEE, 2006, pp. 2766-2769.

[7] H. Wang, D. R. Wade Jr, J. Kam, IR imaging of blood circulation of patients with vascular disease, in: Defense and Security, International Society for Optics and Photonics, 2004, pp. 115-123.

[8] G. Santa Cruz, J. Bertotti, J. Marin, S. Gonzalez, S. Gossio, D. Alvarez, B. Roth, P. Menéndez, M. Pereira, M. Albero, et al., Dynamic infrared imaging of cutaneous melanoma and normal skin in patients treated with BNCT, Applied Radiation and Isotopes 67 (7) (2009) S54-S58.

[9] A. Helmy, M. Holdmann, M. Rizkalla, Application of thermography for non-invasive diagnosis of thyroid gland disease, IEEE Transactions on Biomedical Engineering 55 (3) (2008) 1168-1175.

[10] J.-H. Tan, E. Ng, U. R. Acharya, C. Chee, Infrared thermography on ocular surface temperature: a review, Infrared Physics \& Technology 52 (4) (2009) 97-108.

[11] A. L. Shada, L. T. Dengel, G. R. Petroni, M. E. Smolkin, S. Acton, C. L. Slingluff, Infrared thermography of cutaneous melanoma metastases, Journal of Surgical Research 182 (1) (2013) e9-e14.

[12] M. P. Çetingül, C. Herman, Quantification of the thermal signature of a melanoma lesion, International Journal of Thermal Sciences 50 (4) (2011) 421-431.

[13] M. Bonmarin, F.-A. Le Gal, Lock-in thermal imaging for the early-stage detection of cutaneous melanoma: A feasibility study, Computers in Biology and Medicine 47 (2014) 36-43.

[14] J. R. Keyserlingk, P. Ahlgren, E. Yu, N. Belliveau, M. Yassa, Functional infrared imaging of the breast: Historical perspectives, current application and future considerations, CRC Press, 2006.

[15] R. Hatwar, C. Herman, Inverse method for quantitative characterisation of breast tumours from surface temperature data, International Journal of Hyperthermia 33 (7) (2017) 1-17.

[16] A. Amri, S. H. Pulko, A. J. Wilkinson, Potentialities of steady-state and transient thermography in breast tumour depth detection: A numerical study, Computer Methods and Programs in Biomedicine 123 (2016) 68-80.

[17] K. Ammer, E. Ring, Standard procedures for infrared imaging in medicine, CRC Press, 2006.

[18] T.-Y. Cheng, C. Herman, Analysis of skin cooling for quantitative dynamic infrared imaging of near-surface lesions, International Journal of Thermal Sciences 86 (2014) $175-188$.

[19] M. Stra̧kowska, R. Stra̧kowski, M. Strzelecki, G. De Mey, B. Więcek, Evaluation of perfusion and thermal parameters of skin tissue using cold provocation and thermographic measurements, Metrology and Measurement Systems 23 (3) (2016) 373-381. 
[20] F. Greene, C. Compton, A. Fritz, J. Shah, D. Winchester, Melanoma of the skin, AJCC Cancer Staging Atlas Part V (2006).

[21] W. H. Clark, L. From, E. A. Bernardino, M. C. Mihm, The histogenesis and biologic behavior of primary human malignant melanomas of the skin, Cancer Research 29 (3) (1969) 705-727.

[22] A. Breslow, Thickness, cross-sectional areas and depth of invasion in the prognosis of cutaneous melanoma, Annals of Surgery 172 (5) (1970) 902-908.

[23] A. A. Marghoob, K. Koenig, F. V. Bittencourt, A. W. Kopf, R. S. Bart, Breslow thickness and Clark level in melanoma: support for including level in pathology reports and in American Joint Committee on Cancer staging, Cancer 88 (3) (2000) $589-595$.

[24] J. K. Patel, S. Konda, O. A. Perez, S. Amini, G. Elgart, B. Berman, Newer technologies/techniques and tools in the diagnosis of melanoma, European Journal of Dermatology 18 (6) (2008) 617-631.

[25] D. S. Rigel, J. Russak, R. Friedman, The evolution of melanoma diagnosis: 25 years beyond the ABCDs, CA: A Cancer Journal for Clinicians 60 (5) (2010) 301-316.

[26] J. E. Mayer, S. M. Swetter, T. Fu, A. C. Geller, Screening, early detection, education, and trends for melanoma: current status (2007-2013) and future directions: Part I. Epidemiology, high-risk groups, clinical strategies, and diagnostic technology, Journal of the American Academy of Dermatology 71 (4) (2014) 599.e1-599.e12.

[27] J. E. Mayer, S. M. Swetter, T. Fu, A. C. Geller, Screening, early detection, education, and trends for melanoma: current status (2007-2013) and future directions: Part II. Screening, education, and future directions, Journal of the American Academy of Dermatology 71 (4) (2014) 611.e1-611.e10.

[28] D. Wartman, M. Weinstock, Are we overemphasizing sun avoidance in protection from melanoma?, Cancer Epidemiology and Prevention Biomarkers 17 (3) (2008) 469-470.

[29] R. Marchesini, A. Bono, C. Bartoli, M. Lualdi, S. Tomatis, N. Cascinelli, Optical imaging and automated melanoma detection: questions and answers, Melanoma Research 12 (3) (2002) 279-286.

[30] A. A. Marghoob, L. D. Swindle, C. Z. Moricz, F. A. S. Negron, B. Slue, A. C. Halpern, A. W. Kopf, Instruments and new technologies for the in-vivo diagnosis of melanoma, Journal of the American Academy of Dermatology 49 (5) (2003) 777-797.

[31] E. L. Psaty, A. C. Halpern, Current and emerging technologies in melanoma diagnosis: the state of the art, Clinics in Dermatology 27 (1) (2009) 35-45.

[32] S. Q. Wang, H. Rabinovitz, A. W. Kopf, M. Oliviero, Current technologies for the in vivo diagnosis of cutaneous melanomas, Clinics in Dermatology 22 (3) (2004) $217-222$. 
[33] D. Gutkowicz-Krusin, M. Elbaum, A. Jacobs, S. Keem, A. Kopf, H. Kamino, S. Wang, P. Rubin, H. Rabinovitz, M. Oliviero, Precision of automatic measurements of pigmented skin lesion parameters with a MelaFind(TM) multispectral digital dermoscope, Melanoma Research 10 (6) (2000) 563-570.

[34] A. Bhowmik, R. Repaka, S. C. Mishra, K. Mitra, Analysis of radiative signals from normal and malignant human skins subjected to a short-pulse laser, International Journal of Heat and Mass Transfer 68 (2014) 278-294.

[35] J. March, M. Hand, D. Grossman, Practical application of new technologies for melanoma diagnosis: Part I. noninvasve approaches, Journal of the American Academy of Dermatology 72 (6) (2015) 929-941.

[36] L. Lim, B. Nichols, M. R. Migden, N. Rajaram, J. S. Reichenberg, M. K. Markey, M. I. Ross, J. W. Tunnell, Clinical study of noninvasive in vivo melanoma and nonmelanoma skin cancers using multimodal spectral diagnosis, Journal of Biomedical Optics 19 (11) (2014) 117003.

[37] M. Stücker, I. Horstmann, C. Nüchel, A. Röchling, K. Hoffmann, P. Altmeyer, Blood flow compared in benign melanocytic naevi, malignant melanomas and basal cell carcinomas, Clinical and Experimental Dermatology 24 (2) (1999) 107-111.

[38] J. Iljaž, L. C. Wrobel, M. Hriberšek, J. Marn, The use of Design of Experiments for steady-state and transient inverse melanoma detection problems, International Journal of Thermal Sciences 135 (2019) 256-275.

[39] M. Çetingül, C. Herman, A heat transfer model of skin tissue for detection of lesions: Sensitivity analysis, Phys. Med. Biol 55 (2010) 5933-5951.

[40] C. W. Song, J. G. Rhee, S. H. Levitt, Blood flow in normal tissues and tumors during hyperthermia, Journal of the National Cancer Institute 64 (1) (1980) 119-124.

[41] J. Iljaž, L. C. Wrobel, M. Hriberšek, J. Marn, Numerical modelling of skin tumour tissue with temperature-dependent properties for dynamic thermography, Under review for Computers in Biology and Medicine.

[42] J. M. Luna, R. Romero-Mendez, A. Hernandez-Guerrero, F. Elizalde-Blancas, Procedure to estimate thermophysical and geometrical parameters of embedded cancerous lesions using thermography, Journal of Biomechanical Engineering 134 (3) (2012) 031008-1-031008-9.

[43] A. R. Melo, M. M. S. Loureiro, F. Loureiro, Blood perfusion parameter estimation in tumors by means of a genetic algorithm, Procedia Computer Science 108 (2017) 1384-1393.

[44] P. W. Partridge, L. C. Wrobel, A coupled dual reciprocity BEM/genetic algorithm for identification of blood perfusion parameters, International Journal of Numerical Methods for Heat \& Fluid Flow 19 (1) (2009) 25-38.

[45] M. Paruch, E. Majchrzak, Identification of tumor region parameters using evolutionary algorithm and multiple reciprocity boundary element method, Engineering Applications of Artificial Intelligence 20 (5) (2007) 647-655. 
[46] K. Das, S. C. Mishra, Simultaneous estimation of size, radial and angular locations of a malignant tumor in a 3-D human breast - A numerical study, Journal of Thermal Biology 52 (2015) 147-156.

[47] A. A. Wahab, M. I. M. Salim, M. A. Ahamat, N. A. Manaf, J. Yunus, K. W. Lai, Thermal distribution analysis of three-dimensional tumor-embedded breast models with different breast density compositions, Medical \& Biological Engineering \& Computing 54 (9) (2016) 1363-1373.

[48] J. M. Luna, A. Hernández Guerrero, R. Romero Méndez, J. L. Luviano Ortiz, Solution of the inverse bio-heat transfer problem for a simplified dermatological application: Case of skin cancer, Ingeniería Mecánica, Tecnología y Desarrollo 4 (6) (2014) 219-228.

[49] A. Bhowmik, R. Repaka, Estimation of growth features and thermophysical properties of melanoma within 3-D human skin using genetic algorithm and simulated annealing, International Journal of Heat and Mass Transfer 98 (2016) 81-95.

[50] P. W. Partridge, L. C. Wrobel, An inverse geometry problem for the localisation of skin tumours by thermal analysis, Engineering Analysis with Boundary Elements 31 (10) (2007) 803-811.

[51] F. R. Bueno, P. W. Partridge, An inverse model for locating skin tumours in 3D using the genetic algorithm with the dual reciprocity boundary element method, Latin American Journal of Solids and Structures 10 (6) (2013) 1061-1079.

[52] J. Iljaž, L. Škerget, Blood perfusion estimation in heterogeneous tissue using BEM based algorithm, Engineering Analysis with Boundary Elements 39 (2014) 75-87.

[53] F. S. Bazán, L. Bedin, L. S. Borges, Space-dependent perfusion coefficient estimation in a 2D bioheat transfer problem, Computer Physics Communications 214 (2017) 1830 .

[54] D. Trucu, D. B. Ingham, D. Lesnic, Reconstruction of the space-and time-dependent blood perfusion coefficient in bio-heat transfer, Heat Transfer Engineering 32 (9) (2011) 800-810.

[55] A. Bhowmik, R. Repaka, S. C. Mishra, Thermographic evaluation of early melanoma within the vascularized skin using combined non-Newtonian blood flow and bioheat models, Computers in Biology and Medicine 53 (2014) 206-219.

[56] A. Bhowmik, R. Repaka, R. Mulaveesala, S. C. Mishra, Suitability of frequency modulated thermal wave imaging for skin cancer detection - A theoretical prediction, Journal of Thermal Biology 51 (2015) 65-82.

[57] A. B. C. Silva, J. Laszczyk, L. C. Wrobel, F. L. Ribeiro, A. J. Nowak, A thermoregulation model for hypothermic treatment of neonates, Medical Engineering \& Physics 38 (9) (2016) 988-998.

[58] D. Fiala, K. J. Lomas, M. Stohrer, A computer model of human thermoregulation for a wide range of environmental conditions: the passive system, Journal of Applied Physiology 87 (5) (1999) 1957-1972. 
[59] D. Fiala, G. Havenith, P. Bröde, B. Kampmann, G. Jendritzky, UTCI-Fiala multinode model of human heat transfer and temperature regulation, International Journal of Biometeorology 56 (3) (2012) 429-441.

[60] H. H. Pennes, Analysis of tissue and arterial blood temperatures in the resting human forearm, Journal of Applied Physiology 1 (2) (1948) 93-122.

[61] S. B. Wilson, V. A. Spence, A tissue heat transfer model for relating dynamic skin temperature changes to physiological parameters, Physics in Medicine \& Biology 33 (8) (1988) 895-912.

[62] J. Werner, M. Buse, Temperature profiles with respect to inhomogeneity and geometry of the human body, Journal of Applied Physiology 65 (3) (1988) 1110-1118.

[63] J. W. Mitchell, T. L. Galvez, J. Hengle, G. E. Myers, K. L. Siebecker, Thermal response of human legs during cooling, Journal of Applied Physiology 29 (6) (1970) 859-865.

[64] J. E. Laszczyk, A. J. Nowak, Computational modelling of neonate's brain cooling, International Journal of Numerical Methods for Heat \& Fluid Flow 26 (2) (2016) $571-590$.

[65] J. Iljaž, L. C. Wrobel, M. Hriberšek, J. Marn, Subdomain BEM formulations for the solution of bio-heat problems in biological tissue with melanoma lesions, Engineering Analysis with Boundary Elements 83 (2017) 25-42. 